\title{
A Constraint-Based Hypergraph Partitioning Approach to Coreference Resolution
}

\author{
Emili Sapena* \\ Universitat Politècnica de Catalunya \\ Lluís Padró** \\ Universitat Politècnica de Catalunya \\ Jordi Turmo ${ }^{\dagger}$ \\ Universitat Politècnica de Catalunya
}

This work is focused on research in machine learning for coreference resolution. Coreference resolution is a natural language processing task that consists of determining the expressions in a discourse that refer to the same entity.

The main contributions of this article are (i) a new approach to coreference resolution based on constraint satisfaction, using a hypergraph to represent the problem and solving it by relaxation labeling; and (ii) research towards improving coreference resolution performance using world knowledge extracted from Wikipedia.

The developed approach is able to use an entity-mention classification model with more expressiveness than the pair-based ones, and overcome the weaknesses of previous approaches in the state of the art such as linking contradictions, classifications without context, and lack of information evaluating pairs. Furthermore, the approach allows the incorporation of new information by adding constraints, and research has been done in order to use world knowledge to improve performances.

RelaxCor, the implementation of the approach, achieved results at the state-of-the-art level, and participated in international competitions: SemEval-2010 and CoNLL-2011. RelaxCor achieved second place in CoNLL-2011.

\section{Introduction}

Coreference resolution is a natural language processing (NLP) task that consists of determining which mentions in a discourse refer to the same entity or event. A mention is a referring expression that has an entity or event as a referent. By referring expression we mean noun phrases (NP), named entities (NEs), embedded nouns, and pronouns (all but pleonastic and interrogative ones) whose meaning as a whole is a

\footnotetext{
* TALP Research Center, Universitat Politècnica de Catalunya. E-mail: esapena@lsi .upc . edu.

** TALP Research Center, Universitat Politècnica de Catalunya. E-mail: padro@lsi .upc. edu.

$\dagger$ TALP Research Center, Universitat Politècnica de Catalunya. E-mail: turmo@lsi . upc. edu.
} 
$\left[[\text { FC Barcelona }]_{0} \text { president Joan Laporta }\right]_{1}$ has warned [Chelsea $]_{2}$ off [star striker Lionel Messi $]_{3}$.

Aware of $\left[[\text { Chelsea }]_{2} \text { owner Roman Abramovich }\right]_{4}$ 's interest in [the young Argentine $]_{3}$, [Laporta $]_{1}$ said last night: " $[\mathrm{I}]_{1}$ will answer as always, $[\text { Messi }]_{3}$ is not for sale and [we $]_{0}$ do not want to let $[\text { him }]_{3}$ go."

\section{Figure 1}

Example of coreference resolution. All the mentions are annotated with a subscript indicating their coreference chain. Boldfaced mentions refer to the entity Lionel Messi.

reference to an entity or event in the real world, which is what we call referent. In this article, we do not deal with coreference involving events, and focus only on entity correference.

Coreference chains or entities are groups of referring expressions that have the same referent. Thus, a coreference chain is formed by all mentions in a discourse that refer to the same real entity. Given an arbitrary text as input, the goal of a coreference resolution system is to find all the coreference chains. A partial entity is a set of mentions considered coreferential during resolution.

Figure 1 shows the mentions of a newspaper article and their corresponding coreference chains. Note that the difficulty of coreference resolution lies in the variety of necessary knowledge sources. For instance, morphological and syntactic analysis is needed to detect mentions, and semantic/world knowledge to know that Messi is a star striker and a young Argentine.

Coreference resolution is a mandatory step in order to understand natural language. In this sense, dealing with such a problem becomes important for tasks in which the higher their comprehension of the discourse, the better such systems will performtasks such as machine translation (Peral, Palomar, and Ferrández 1999), question answering (Morton 2000), summarization (Azzam, Humphreys, and Gaizauskas 1999), and information extraction.

One of the possible directions to follow in coreference resolution research is the incorporation of new information such as world knowledge and discourse coherence. In some cases, this information cannot be expressed in terms of pairs of mentions-that is, it is information that involves either several mentions at once or partial entities. Furthermore, an experimental approach in this field should overcome the weaknesses of previous state-of-the-art approaches, such as linking contradictions, classifications without context, and a lack of information when evaluating pairs.

This article presents an approach for coreference resolution based on constraint satisfaction that represents the problem in a hypergraph and solves it by relaxation labeling. One of the main goals of developing such an approach is the incorporation of world knowledge and discourse coherence in order to improve performance while addressing the problems mentioned previously.

The article is structured as follows. Section 2 summarizes the state of the art of machine learning approaches to coreference resolution, highlighting their most relevant parts with their corresponding issues. Section 3 defines our proposed approach and Section 4 provides details about the implementation and the training methods. The experiments and error analysis are described in Section 5. Section 6 presents our approach to incorporate world knowledge in order to improve coreference resolution performance. Experiments and a detailed error analysis are also included. Finally, we discuss the conclusions of this article in Section 7. 


\section{Coreference Resolution: State of the Art}

In this section we summarize the main machine-learning-based approaches to coreference resolution. For a wider study, we refer the reader to Mitkov (2002).

A coreference resolution system receives plain text as input, and returns the same text with coreference annotations as output. Most existing coreference resolution systems can be considered instances of this general process, which consists of three main steps: mention detection, characterization of mentions, and resolution (see Figure 2).

The first step is the detection of mentions, where text processing is needed in order to find the boundaries of the mentions in the input text. Next, in the second step, the identified mentions are characterized by gathering all the available knowledge about them and their possible compatibility. Typically, machine learning systems introduce all the knowledge by means of feature functions. Finally, the resolution itself is performed in the third step. A generalization of the inner architecture of the resolution step is difficult given the diversity of approaches and algorithms used for resolution. Even so, the diverse approaches in current systems have at least two main processes in the resolution: classification and linking.

- Classification. This process evaluates the compatibility of elements in order to corefer. The elements can be mentions or partial entities. A typical implementation is a binary classifier that assigns class $\mathrm{CO}$ (coreferential) or NC (not coreferential) to a pair of mentions. It is also very typical to use confidence values or probabilities associated with the class. Classifiers can also use rankers and constraints.

- $\quad$ Linking. The linking process links mentions and partial entities in order to form the final entities. This process may range from a simple heuristic, such as single-link, to an elaborate algorithm such as clustering or graph partitioning. The input of the linking process includes the output of the classification process: classes and probabilities.

\subsection{Classification Models}

The models found in the state of the art for the classification process are: mention pairs, rankers, and entity-mention.

Mention pairs. Classifiers based on the mention-pair model determine whether two mentions corefer or not. To do so, a feature vector is generated for a pair of mentions using a set of features. Given these features as input, the classifier returns a class: $\mathrm{CO}$ (coreferent), or NC (not coreferent). In many cases, the classifier also returns a confidence value about the decision taken. The class and the confidence value of each

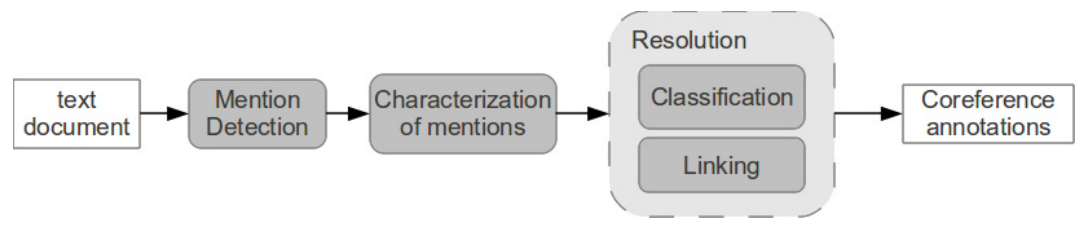

Figure 2

Architecture of a coreference resolution system. 


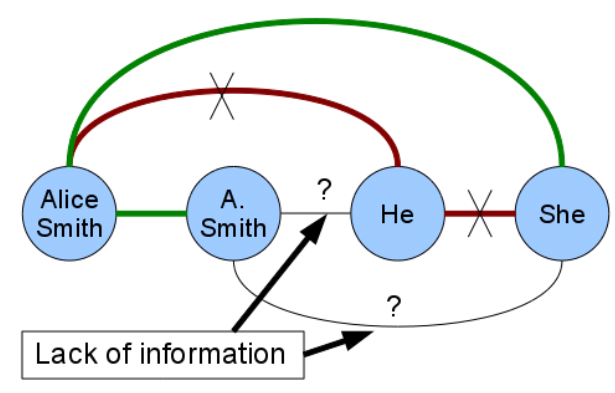

\section{Figure 3}

A pairwise classifier does not have enough information to classify pairs (A. Smith, he) and $(A$. Smith, she).

evaluated pair of mentions will be taken into account by the linking process to obtain the final result.

The mention-pair model has two main weaknesses: a lack of contextual information and contradictions in classifications. Figure 3 shows an example of lack of information. The figure is a representation of a document with four mentions (Alice Smith, A. Smith, he, she). The edges between mentions represent the classification in a mention-pair model; green means that the classifier returns the $\mathrm{CO}$ class, and red (also marked with an X) returns the NC class. In this case, the lack of information is due to the impossibility of determining the gender of $A$. Smith. Next, Figure 4 shows a possible scenario with contradictions. In this scenario, the classifier has determined that the pairs (A. Smith, he) and (A. Smith, she) corefer, which causes contradictions when generating the final coreference chains given that the pairs (Alice Smith, he) and (he, she) do not corefer.

Rankers. The rankers model overcomes the lack of contextual information found using mention-pairs. Instead of directly considering whether $m_{i}$ and $m_{j}$ corefer, more perspective can be achieved by looking for the best candidate from a group of mentions to corefer with an active mention. Rankers can still fall in contradictions, however, and need to rely on the linking process to solve that.

Entity-mention. The entity-mention model classifies a partial entity and a mention, or two partial entities, as coreferent or not. In some models, a partial entity even has its

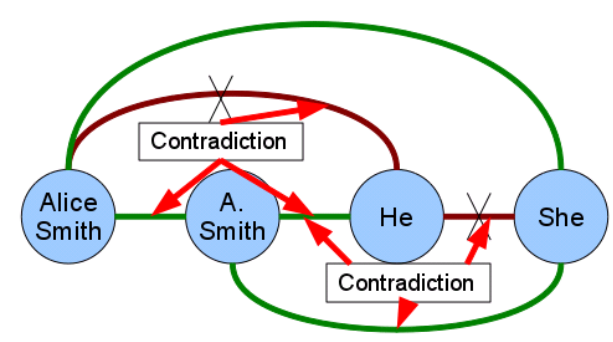

Figure 4

Green edges mean that both mentions corefer, and red edges mean the opposite. An independent classification of $(A$. Smith, he) and (A. Smith, she) produces contradictions. 
own properties or features defined in the model in order to be compared with the mentions. Due to the information that a partial entity gives to the classifier, in most cases this model overcomes the lack of information and contradiction problems of the mention-based models. For example, a partial entity may include the mentions Alice Smith and A. Smith, whose genders are "female" and "unknown" respectively. In this case, the partial entity is more likely to be linked with the subsequent mention she than with he (Figures 3 and 4). The features used for entity-mention models are almost the same as those used for mention-based models. The only difference is that the value of an entity feature is determined by considering the particular values of the mentions belonging to it.

\subsection{Resolution}

The coreference resolution engines in the state of the art can be classified into three paradigms depending on their resolution process (i.e., combinations of classification and linking processes):

- $\quad$ Backward search approaches classify mentions with previous ones, looking for the best antecedents. In this case, the linking step is typically an heuristic that links mention pairs classified as positive (single-link).

- Two-step approaches perform the resolution in two separate steps. The first step is to classify all of the elements, and then the second step is a linking process using algorithms such as graph partitioning or clustering to optimize the results given the classification output.

- One-step approaches directly run the linking process while classification is performed on-line. In this manner, mention-group and entity-mention models can be easily incorporated.

Figure 5 summarizes the classification of several systems in the state of the art, up to 2011. Recently, the CoNLL-2012 shared task (Pradhan et al. 2012) offered an evaluation framework similar to that of CoNLL-2011. The second column specifies which resolution step is used. The third column shows the classification model used by the system, and the fourth column identifies the algorithm followed in the linking process.

More details about supervised machine learning systems can be found in $\mathrm{Ng}$ (2010).

\section{A Constraint-Based Hypergraph Partitioning Approach to Coreference Resolution}

One of the possible directions to follow in coreference resolution research is the incorporation of new information such as world knowledge and discourse coherence. In some cases, this information cannot be expressed in terms of pairs of mentions, that is, it is information that involves either several mentions at once or partial entities. Therefore, an experimental approach in this field needs the expressiveness of the entity-mention model as well as the mention-pair model in order to use the most typical mention-pair features. Furthermore, such an approach should overcome the weaknesses of previous state-of-the-art approaches, such as linking contradictions, classifications without context, and a lack of information when evaluating pairs. Also, the approach would be more flexible if it could incorporate knowledge both automatically and manually. 


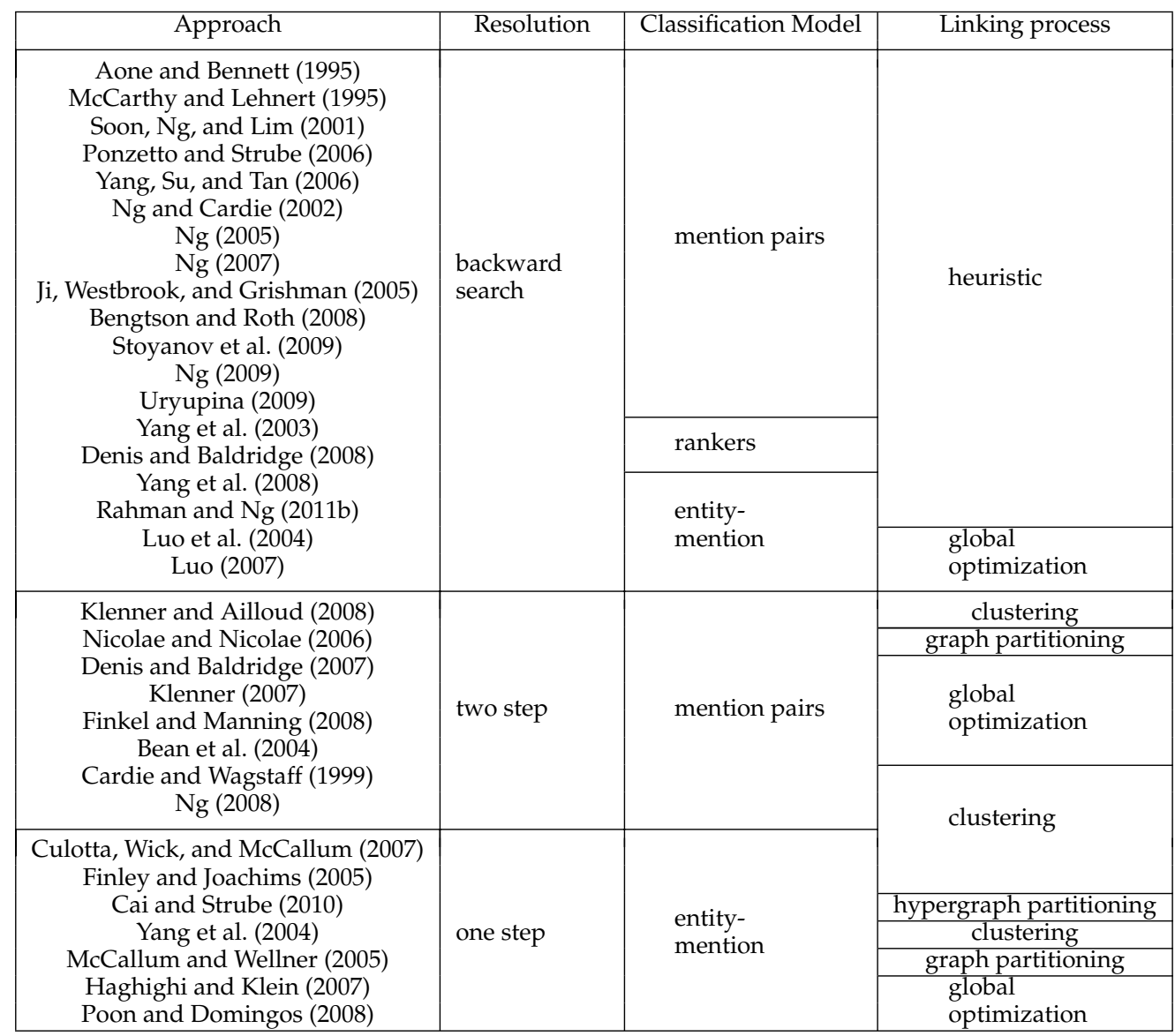

Figure 5

A classification of coreference resolution approaches in state-of-the-art machine-learning systems.

Given these prerequisites, we define an approach based on constraint satisfaction that represents the problem in a hypergraph and solves it by relaxation labeling, reducing coreference resolution to a hypergraph partitioning problem with a given set of constraints. The main strengths of this system are:

- $\quad$ Modeling the problem in terms of hypergraph partitioning avoids linking contradictions and errors caused by a lack of information or context.

- Constraints are compatible with the mention-pair and entity-mention models, which let us incorporate new information. Moreover, constraints can be both automatically learned and manually written.

- Relaxation labeling is an iterative algorithm that performs function optimization based on local information. It first determines the entities of the mentions in which it has more confidence, mainly solving the problem of lack of information for some pairs and the lack of context. The iterative resolution facilitates the use of the entity-mention model.

The rest of this section describes the details of the approach. Section 3.1 describes the problem representation in a (hyper)graph. Next, Section 3.2 explains how the 
knowledge is represented as a set of constraints, and Section 3.3 explains how attaching influence rules to the constraints means that the approach incorporates the entitymention model. Finally, Section 3.4 describes the relaxation labeling algorithm used for resolution.

\subsection{Graph and Hypergraph Representations}

The coreference resolution problem consists of a set of mentions that have to be mapped to a minimal collection of individual entities. By representing the problem in a hypergraph, we are reducing coreference resolution to a hypergraph partitioning problem. Each partition obtained in the resolution process is finally considered an entity.

The document mentions are represented as vertices in a hypergraph. Each of these vertices is connected by hyperedges to other vertices. Hyperedges are assigned a weight that indicates the confidence that adjacent mentions corefer. The larger the hyperedge weight in absolute terms, the more reliable the hyperedge. In the case of the mention-pair model, the problem is represented as a graph where edges connect pairs of vertices.

Let $G=G(V, E)$ be an undirected hypergraph, where $V$ is a set of vertices and $E$ is a set of hyperedges. Let $\mathbf{m}=\left(m_{1}, \ldots, m_{n}\right)$ be the set of mentions of a document with $n$ mentions to resolve. Each mention $m_{i}$ in the document is represented as a vertex $v_{i} \in V$. A hyperedge $e_{g} \in E$ is added to the hypergraph for each group $(g)$ of vertices $\left(v_{0}, \ldots, v_{N}\right)$ affected by a constraint, as shown in Figure 6 . The subset of hyperedges that incide on $v_{i}$ is $E\left(v_{i}\right)$.

A subset of constraints $C_{g} \subseteq C$ restricts the compatibility of a group of mentions. $C_{g}$ is used to compute the weight value of the hyperedge $e_{g}$. Let $w\left(e_{g}\right) \in W$ be the weight of the hyperedge $e_{g}$ :

$$
w\left(e_{g}\right)=\sum_{k \in C_{g}} \lambda_{k}
$$

where $\lambda_{k}$ is the weight associated with constraint $k$. The graph representing the mentionpair model is a subcase of the hypergraph where $|g|=2$. Figure 7 illustrates a graph. For simplicity, in the case of the mention-pair model, an edge between $m_{i}$ and $m_{j}$ is called $e_{i j}$. In addition, sometimes $w_{i j}$ is used instead of $w\left(e_{i j}\right)$.

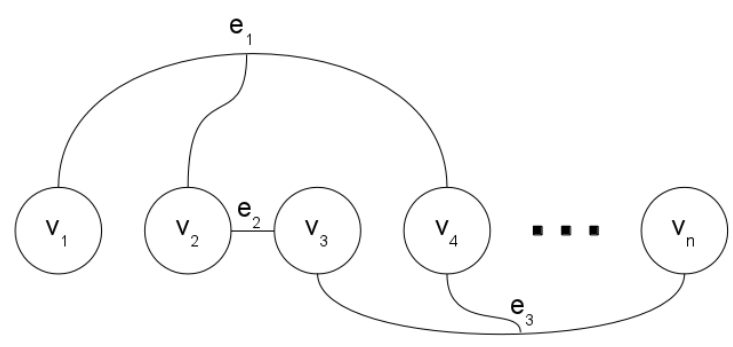

Figure 6

Example of hypergraph representing the mentions of a document connected by hyperedges (mention-group model). 


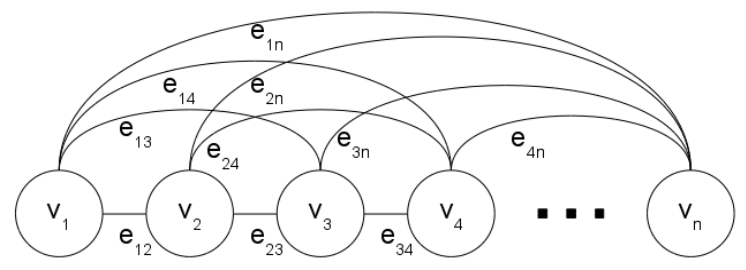

Figure 7

Example of graph representing the mentions of a document connected by edges (mention-pair model).

DIST_SEN_1(0,1) \& GENDER_YES(0,1) \& $\neg$ FIRST(0) \&

MAXIMALNP(0) \& MAXIMALNP(1) \&

SRL_ARG_0(0) \& SRL_ARG_0(1) \&

TYPE_P(0) \& TYPE_P(1)

Figure 8

Example of a mention-pair constraint $(N=2)$.

DIST_SEN_1(0,1) \& DIST_SEN_1(1,2) \&

AGREEMENT_YES $(0,1,2) \&$ ALIAS_YES(0,2) \&

SRL_ARG_0(0) \& SRL_ARG_0(1) \& SRL_ARG_0(2) \&

TYPE_E(0) \& TYPE_S(1) \& TYPE_E(2)

\section{Figure 9}

Example of a mention-group constraint $(N=3)$.

\subsection{Constraints as Knowledge Representation}

In this approach, knowledge is a set of weighted constraints where each constraint contributes a piece of information that helps to determine the coreferential relations between mentions. A constraint is a conjunction of feature-value pairs that are evaluated over all the pairs or groups of mentions in a document. When a constraint applies to a set of mentions, a corresponding hyperedge is added to the hypergraph, generating the representation of the problem explained in Section 3.1 (Figure 6).

Let $N$ be the order of a constraint, that is, the number of mentions expected by the constraint $(|g|)$. A pair constraint has order $N=2$, and a group constraint has $N>2$. The mentions evaluated by a constraint are numbered from 0 to $N-1$ in the order they are found in the document.

Figures 8 and 9 show examples of constraints for $N=2$ and $N=3$, respectively. The constraint in Figure 8 requires that: The distance between the mentions is just one sentence, their genders match, $m_{0}$ is not the first mention of its sentence, $m_{0}$ is a maximal NP (the next parent node in the syntactic tree is the sentence itself), $m_{1}$ also is a maximal NP, both mentions are ARG0 in semantic role labeling, and both mentions are pronouns. ${ }^{1}$ The constraint in Figure 9 applies to three mentions and requires that: The distance between consecutive mentions is one sentence, all three mentions agree in both gender and number, $m_{0}$ and $m_{2}$ are aliases, all three mentions are ARG0 in their respective sentences, and $m_{0}$ and $m_{2}$ are named entities and $m_{1}$ is

1 The argument system used is due to PropBank (Kingsbury and Palmer 2003). 
a common $\mathrm{NP}^{2}$ There are many examples of negative constraints, that is, constraints that restrict mentions from being in the same entity. For instance GENDER_NO $(0,1)$ \& TYPE_P(0) \& TYPE_P(1) expresses that $m_{1}$ and $m_{0}$ are pronouns and do not match in gender.

Each constraint has a weight that determines the hyperedge weight of the hypergraph (see Equation (1)). A constraint weight is a value that, in absolute terms, reflects the confidence of the constraint. Moreover, this weight is signed, and the sign indicates whether the adjacent mentions corefer (positive) or not (negative). The use of negative information is not very extensive in state-of-the-art systems, but given the hypergraph representation of the problem, where most of the mentions are interconnected, the negative weights contribute information that cannot be obtained using only positive weights. Moreover, in our experiments, the use of negative weights accelerates the convergence of the resolution algorithm. The training process that determines the weight of each constraint is explained in Section 4.3.

\subsection{Entity-Mention Model Using Influence Rules}

We have explained how groups of mentions satisfying a constraint are connected by hyperedges in the hypergraph. This section explains how the entity-mention model is definitively incorporated to our constraint-based hypergraph approach. The entitymention model takes advantage of the concept of an entity during the resolution process. This means that each mention belongs to an entity during resolution, and this information can be used to make new decisions.

In order to incorporate the entity-mention model into our approach, we define the influence rule, which is attached to a constraint. An influence rule expresses the conditions that the mentions must meet during resolution before the influence of the constraint takes effect.

An influence rule consists of two parts: condition and action.

- The condition of an influence rule is a conjunction of coreference relations that the mentions must satisfy before the constraint has influence. This condition is specified by joining mentions into groups, where each group represents a partial entity specified by a subscript. For instance, $(0,1)_{A},(2)_{B}$ means that mentions 0 and 1 belong to entity $A$ and mention 2 belongs to entity $B(A \neq B)$.

- The action of an influence rule defines the desired coreference relation and determines which mentions are influenced. It is expressed in the same terms as the condition, specifying the mentions that are influenced and the entity to which they should belong. For instance, an action can be $(3)_{B}$. This action indicates that mention 3 is influenced in order to belong to entity $B$.

Figure 10 shows an example of an $N=4$ constraint with an influence rule attached. The constraint specifies the feature functions that the involved mentions must meet, such as semantic role arguments, sentence distances, and agreements. The influence rule then determines that when mentions 0 and 2 belong to the same entity, and mention 1

2 Feature functions used in our experiments are explained in detail in Section 4.2. 


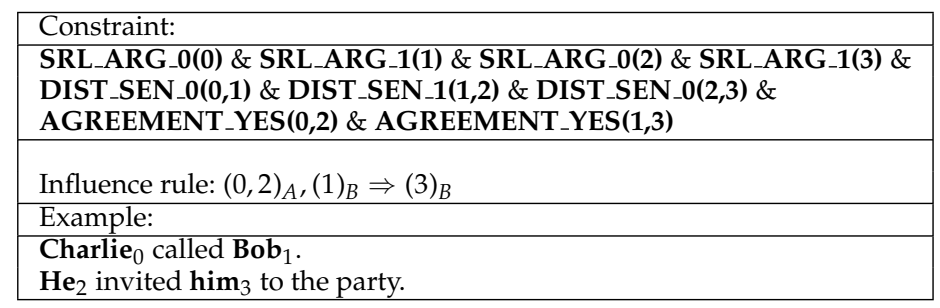

\section{Figure 10}

Artificial example of an entity-mention constraint. It takes advantage of the partial entities during resolution. If mentions 0 and 2 tend to corefer, the structure indicates that mentions 1 and 3 may corefer in a different entity.

belongs to a different entity, mention 3 is influenced in order to belong to the same entity as mention 1 . This figure also contains some text to help understand why this kind of constraint may be useful. A mention-pair approach could easily make the mistake of classifying mentions 2 and 3 as coreferent. This is an example of introducing information about discourse coherence using an entity-mention model.

In order to retain consistency with the mention-pair model, all the constraints used in this approach are assigned a default influence rule that depends on the sign of the edge weight. In the case that the weight is positive, the last mention is influenced to belong to the same entity as the first mention, and a negative weight causes the opposite. Figure 11 shows the default influence rules for mention-pair constraints with both positive and negative weights.

Note that when influence rules are used, a hyperedge is added for each subset of constraints that applies to the same group of mentions and has the same influence rule. In the case that some constraints apply to the same group of mentions but have different influence rules, a hyperedge is added to the graph for each influence rule. Therefore, in Equation (1), $C_{g} \subseteq C$ refers to the constraints that apply to the group and share the same influence rule.

\subsection{Relaxation Labeling}

Relaxation is a generic name for a family of iterative algorithms that perform function optimization based on local information. They are closely related to neural nets and gradient steps. Relaxation labeling has been successfully used in engineering fields to solve systems of equations, in Artificial Intelligence for computer vision (Rosenfeld, Hummel, and Zucker 1976), and in many other AI problems. The algorithm has also been widely used to solve NLP problems such as part-of-speech tagging (Padró 1998), chunking, knowledge integration, semantic parsing (Atserias 2006), and opinion mining (Popescu and Etzioni 2005).

\begin{tabular}{|l|c|c|}
\hline Description & Conditions & Action \\
\hline $\begin{array}{l}\text { Default influence rule for a } \\
\text { mention-pair constraint (positive weight) }\end{array}$ & $(0)_{A}$ & $(1)_{A}$ \\
\hline $\begin{array}{l}\text { Default influence rule for a } \\
\text { mention-pair constraint (negative weight) }\end{array}$ & $(0)_{A}$ & $(1)_{B}$ \\
\hline $\begin{array}{l}\text { Example of an influence rule for an } \\
\text { entity-mention constraint }\end{array}$ & $(0,2)_{A},(1)_{B}$ & $(3)_{B}$ \\
\hline
\end{tabular}

Figure 11

Default influence rules for mention-pair constraints. 


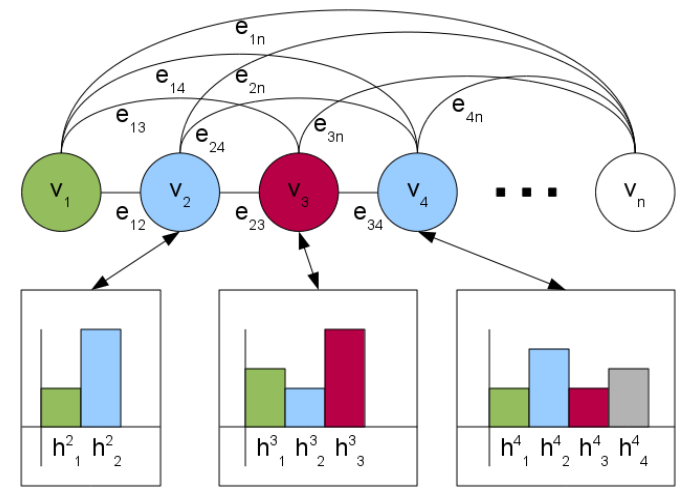

Figure 12

Representation of Relax solving a graph. The vertices representing mentions are connected by weighted edges $e_{i j}$. Each vertex has a vector $h^{i}$ of probabilities to belong to different partitions. The figure shows $h^{2}, h^{3}$, and $h^{4}$.

Relaxation labeling (Relax) solves our weighted constraint-based hypergraph partitioning problem by dealing with (hyper)edge weights as compatibility coefficients. ${ }^{3}$ In this manner, each vertex is assigned to a partition satisfying as many constraints as possible. In each step, the algorithm updates the probability of each vertex belonging to a partition. This update is performed by transferring the probabilities of adjacent vertices proportional to the edge weights.

Let $V=\left\{v_{1}, v_{2}, \ldots, v_{n}\right\}$ be a set of variables. In our approach, each vertex $\left(v_{i}\right)$ in the hypergraph is a variable in the algorithm. Let $L_{i}$ be the number of different labels that are possible for $v_{i}$. The possible labels of each variable are the partitions that the vertex can be assigned. Note that the number of partitions (entities) in a document is a priori unknown, but it is at most the number of vertices (mentions) because, in an extreme case, each mention in a document could refer to a different entity. Therefore, a vertex with index $i$ can be in the first $i$ partitions (i.e., $L_{i}=i$ ).

The aim of the algorithm is to find a weighted labeling such that global consistency is maximized. A weighted labeling is a weight assignment for each possible label of each variable: $\mathbf{H}=\left(\mathbf{h}^{1}, \mathbf{h}^{2}, \ldots, \mathbf{h}^{n}\right)$, where each $\mathbf{h}^{i}$ is a vector containing a weight for each possible label of $v_{i}$; that is, $\mathbf{h}^{i}=\left(h_{1}^{i}, h_{2}^{i}, \ldots, h_{L_{i}}^{i}\right)$. As relaxation is an iterative process, these weights (of between 0 and 1) vary in time. We denote the probability for label $l$ of variable $v_{i}$ at time step $t$ as $h_{l}^{i}(t)$, or simply $h_{l}^{i}$ when the time step is not relevant. Note that the label assigned to a variable at the end of the process is the one with the highest weight $\left(\max \left(\mathbf{h}^{i}\right)\right)$. Figure 12 shows an example.

Maximizing global consistency is defined as maximizing the average support for each variable, which is defined as the weighted sum of the support received by each of its possible labels- that is, $\sum_{l=1}^{L_{i}} h_{l}^{i} \times S_{i l}$, where $S_{i l}$ is the support received by that pair from the context.

The support for a variable-label pair $\left(S_{i l}\right)$ expresses the compatibility of the assignment of label $l$ to variable $v_{i}$ compared with the labels of neighboring variables, according to the edge weights. Although several support functions may be used (Torras

\footnotetext{
3 For the rest of this section, there is no distinction between edges and hyperedges.
} 
1989), we chose the following (Equation (2)), which defines the support as the sum of the influences of the incident edges.

$$
S_{i l}=\sum_{e \in E\left(v_{i}\right)} \operatorname{Inf}(e)
$$

where $\operatorname{Inf}(e)$ is the influence of edge $e$. The influence of an edge is defined by its weight and the influence rules attached to the constraints involved with this edge (see Section 3.3). An influence rule determines how the current probabilities for the same label of adjacent vertices $\left(h_{l}^{j}\right)$ are combined.

The pseudo-code for the relaxation algorithm can be found in Figure 13. It consists of the following steps:

1. Start with a random labeling, or with a better-informed initial state.

2. For each variable, compute the support that each label receives from the current weights of adjacent variable labels following Equation 2.

3. Normalize support values between -1 and 1 . Supports are divided by a ScaleFactor. In case that after that a support is higher than 1 or -1 then its value is cutted to 1 or -1 , respectively. Given that constraint weights are between 1 and -1 and a group of mentions is not generally affected by more than 10 constraints, ScaleFactor is empirically set to 8 in our experiments.

4. Update the weight of each variable label according to the support obtained by each of them (that is, increase weight for labels with high support [greater than zero], and decrease weight for those with low support [less than zero]) according to the update function:

$$
h_{l}^{i}(t+1)=\frac{h_{l}^{i}(t) \times\left(1+S_{i l}\right)}{\sum_{k=1}^{L_{i}} h_{k}^{i}(t) \times\left(1+S_{i k}\right)}
$$

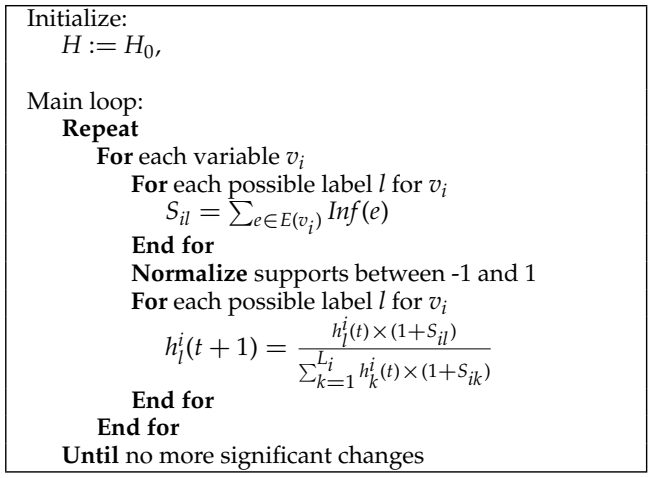

Figure 13

Relaxation labeling algorithm. 
There are many functions that can be used to calculate the support (Torras 1989). The one we chose was also used by Padró (1998) and Màrquez, Padró, and Rodríguez (2000).

5. Iterate the process until the convergence criterion is met. The usual criterion is to wait for no more changes in an iteration, or a maximum change below some epsilon parameter (Equation (4)). But there is also a maximum number of iterations where the process is stopped. This number is a constant and does not depend on the size of the document.

$$
\max \left(h_{l}^{i}(t+1)-h_{l}^{i}(t)\right) \leq|\epsilon| \quad \forall i, l
$$

Each combination of labels for the graph vertices is a partitioning $(\Omega)$. The resolution process searches the partitioning $\Omega^{*}$ which optimizes the goodness function $F(\Omega, W)$, which depends on the edge weights $W$. In this manner, $\Omega^{*}$ is optimal if:

$$
F\left(\Omega^{*}, W\right) \geq F(\Omega, W), \forall \Omega
$$

A partitioning $\Omega$ is directly obtained from the weighted labeling $\mathbf{H}$ assigning to each variable the label with maximum probability. The supports and the weighted labeling depend on the edge weights (Equation (2)). To satisfy Equation (6) is equivalent to satisfying Equation (5). Many studies have been done towards the demonstration of the consistency, convergence, and cost reduction advantages of the relaxation algorithm (Rosenfeld, Hummel, and Zucker 1976; Hummel and Zucker 1983; Pelillo 1997). For instance, Hummel and Zucker (1983) prove that maximizing average consistency (left-hand-side term of Equation (6) produces labelings satisfying Equation (5) when only binary constraints are used. Although there is no formal proof for higher order constraints, the presented algorithm (that forces a stop after a number of iterations) has proven useful for practical purposes in our case.

$$
\sum_{l=1}^{L_{i}} h_{l}^{* i} \times S_{i l} \geq \sum_{l=1}^{L_{i}} h_{l}^{i} \times S_{i l} \quad \forall \mathbf{h}, \forall i
$$

Note that because the weight update for each label is independent of the others, the algorithm can be straightforward parallelized. In the following, there are some examples of the Relax implementation of the edge influences $(\operatorname{Inf}(e))$ given the influence rules attached to the constraints.

The simplest example is when mention $m_{0}$ has a direct influence over mention $m_{1}$. The influence rule attached to the constraint is $(0)_{A} \Rightarrow(1)_{A}$. This is determined by Equation (7) and is the kind of influence used in the mention-pair model.

$$
\operatorname{Inf}(e)=w(e) \times h_{l}^{0}
$$

The next example requires that mention $m_{0}$ and mention $m_{1}$ tend to corefer during the resolution in order to influence mention $m_{2}$. The influence rule is $(0,1)_{A} \Rightarrow(2)_{A}$. In this case, the influence of the edge representing this influence rule is given by Equation (8). Mentions $m_{0}$ and $m_{1}$ are tending to corefer (belong to the same entity: $l$ ) when their values for label $l$ are tending to 1 (and the other labels are tending to 0 ). In this case, multiplying $h_{l}^{0}$ and $h_{l}^{1}$ achieves a value close to 1 , and the influence is almost 


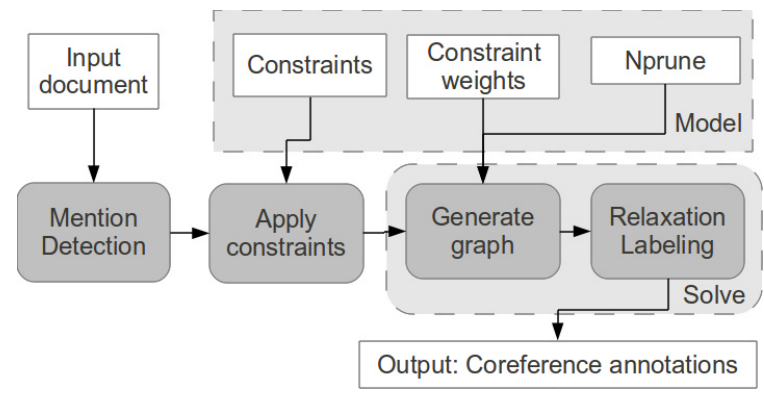

\section{Figure 14}

RELAXCOR resolution process.

the weight of the edge. In other cases when the coreference between $m_{0}$ and $m_{1}$ is not clear (or they are clearly not coreferent), at least one of the values of $h_{l}^{0}$ and $h_{l}^{1}$ is not close to 1 and the value of their product rapidly decreases, so the influence of the edge also decreases.

$$
\operatorname{Inf}(e)=w(e) \times h_{l}^{0} \times h_{l}^{1}
$$

Following the previous example, now suppose that in order for $m_{0}$ to influence $m_{2}$ it is required that $m_{1}$ does not belong to the same entity as $m_{0}$. In this case, $h_{l}^{1}$ is negated using its complementary value $\left(1-h_{l}^{0}\right)$, as is shown in Equation (9). The corresponding influence rule is $(0)_{A},(1)_{B} \Rightarrow(2)_{A}$.

$$
\operatorname{Inf}(e)=w(e) \times h_{l}^{0} \times\left(1-h_{l}^{1}\right)
$$

The complexity of the influence rules can be increased arbitrarily and, theoretically, any number of mentions and entities can be involved. This last example (Equation (10)) shows how to represent $(0,2)_{A},(1)_{B} \Rightarrow(3)_{B}$, an influence rule requiring $m_{0}$ and $m_{2}$ to belong to the same entity, while $m_{1}$ belongs to a different one in order to influence $m_{3}$.

$$
\operatorname{Inf}(e)=w(e) \times h_{l}^{1} \times\left(1-h_{l}^{0} \times h_{l}^{2}\right)
$$

\section{RelaxCor}

RELAXCOR is the coreference resolution system implemented in this work to perform experiments and test the approach explained in Section 3. This section explains the implementation and training methods, before the experiments and error analysis are presented in the following sections. RELAXCOR is programmed in Perl and $\mathrm{C}++$, is open source, and is available for download from our research group's Web site. ${ }^{4}$

The resolution process of RELAXCOR is shown in Figure 14. First, the mention detection system determines the mentions of the input document and their boundaries. The mention detection system is explained in Section 4.1. Alternatively, true mentions can be used when available, allowing this step to be skipped. Next, for each pair or group of mentions (depending on the model), the set of feature functions calculate their values, and the set of model constraints is applied. The set of feature functions used

4 http://nlp.lsi.upc.edu. 
by RELAXCOR and its knowledge sources are explained in Section 4.2. A (hyper)graph is then generated using the applied constraints and their weights. Finally, relaxation labeling is executed to find the partitioning that maximizes constraint satisfaction.

The training and development processes used in this work are described in Sections 4.3 and 4.4. The former explains the method for training the mention-pair model, and the latter concerns the entity-mention model.

\subsection{Mention Detection}

RELAXCOR includes a mention detection system that uses part-of-speech and syntactic information. Syntactic information may be obtained from dependency parsing or constituent parsing. The system extracts one candidate mention for every:

- $\quad$ Noun phrase (NP).

- Pronoun.

- $\quad$ Named Entity.

- Capitalized common noun or proper name that appear two or more times in the document. For instance, the NP an Internet business is a mention, but also Internet is added in the case that the word is found once again in the document.

The head of every candidate mention is then determined using part-of-speech tags and a set of rules from Collins (1999) when constituent parsing is used, or using dependency information otherwise. In case some NPs share the same head, the larger $\mathrm{NP}$ is selected and the rest are discarded. Also, mention repetitions with exactly the same boundaries are discarded. Note that a mention detection system in pipeline configuration with the resolution process acts as a filter and the main objective at this point is to achieve as much recall as possible.

\subsection{Knowledge Sources and Features}

The system gathers knowledge using a set of feature functions that interpret and evaluate the input information according to some criteria. Given a set of mentions numbered from 0 to $N-1$ following the order found in the document, each feature function evaluates their compatibility in a specific aspect. RELAXCOR includes features from all linguistic layers: lexical, syntactic, morphological, and semantic. Moreover, some structural features of the discourse have also been used, such as distances, quotes, and sentential positions. A feature function with only one argument indicates that it offers information about only one mention. For example, REFLEXIVE (0) indicates that mention 0 is a reflexive pronoun. Figure 15 shows an exhaustive list of the features used and a brief description of each one.

We use decision trees for constraint acquisition (see Section 4.3.2). Because the use of binary features favors a better performance in this type of learning (Rounds 1980; Safavian and Landgrebe 1991), all of the used feature functions are binary. The original sources that had a list of possible values have been binarized by a set of feature functions that each represent a different value. Even in numerical cases, there is a set of binary features representing the most important specific values, and the rest are placed in ranges. 


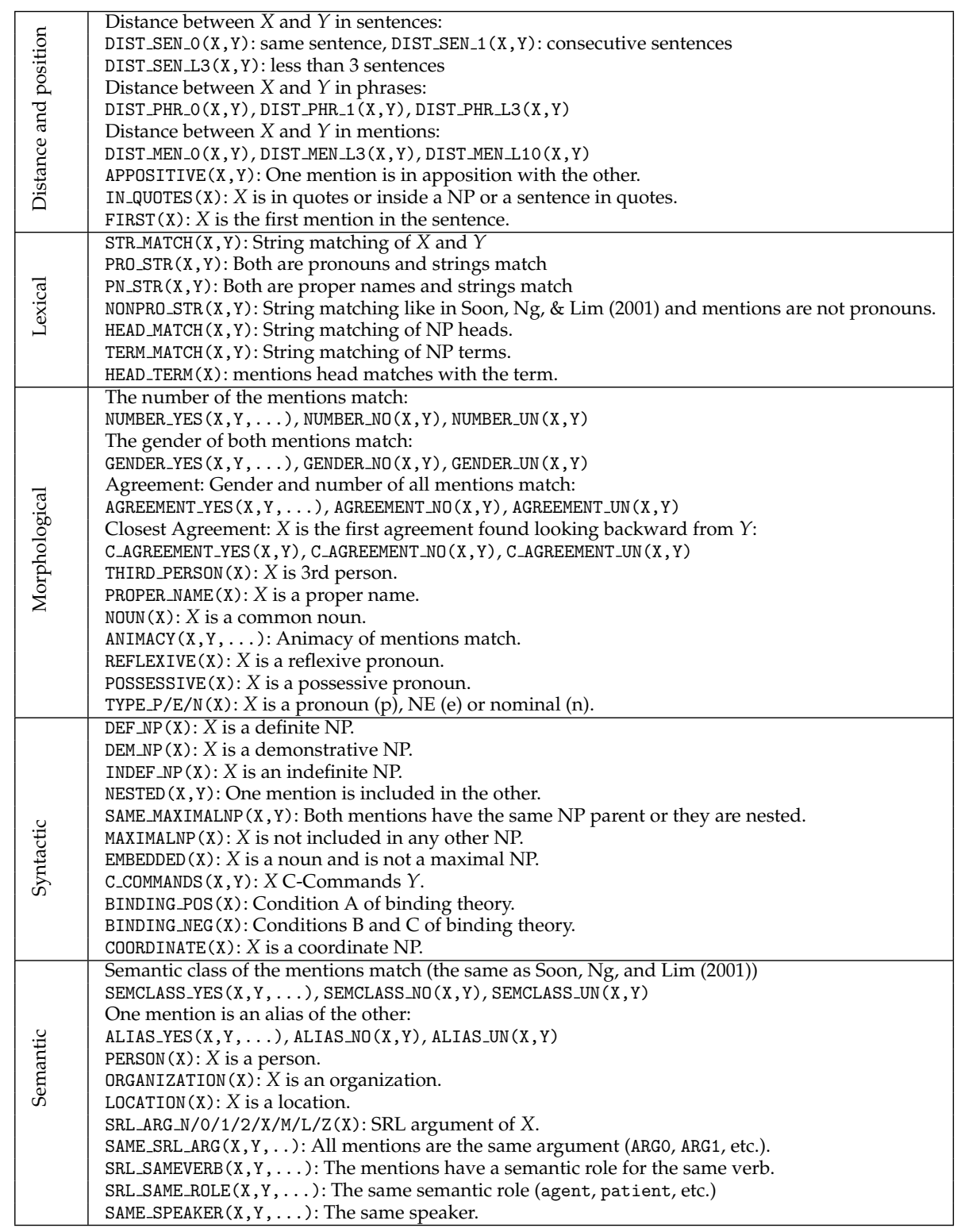

Figure 15

Feature functions used by RELAXCOR.

\subsection{Training and Development for the Mention-Pair Model}

This section describes the training and development process for the implementation of RELAXCOR using the mention-pair model and the graph representation. The training process applies a machine learning algorithm over the training data to obtain a set of constraints. A weight is then assigned to each constraint, taking into account the precision of the constraint finding coreferent mentions.

A machine learning process is applied to obtain the set of constraints. Constraints can also be added writing them by hand. Adding manual constraints is expensive, 


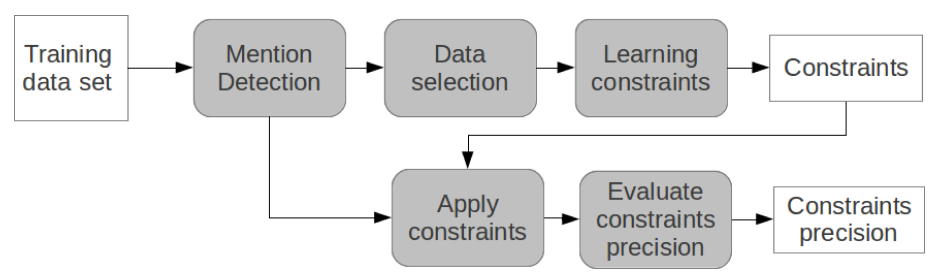

\section{Figure 16}

RELAXCOR training process.

however, given that it takes a group of linguistic experts many hours devoted to this task. An alternative option is to use constraints from other coreference resolution systems, such as the ones used in Lee et al. (2011). Our experiments are based on automatically learned constraints.

Figure 16 shows the training process. First, a data selection process unbalances the training data set and then a machine learning process obtains the constraints. The learned constraints are then applied to the training data set and their precision is evaluated. The precision of each constraint determines its weight. The development process optimizes two parameters-balance and $N_{\text {prune }}$-in order to achieve maximum performance given a measure for the task. Figure 17 shows the development process.

4.3.1 Data Selection. Generating an example for each possible pair of mentions in the training data produces an unbalanced data set in which more than $99 \%$ of the examples are negative (not coreferent). This bias towards negative examples makes the task of the machine learning algorithms difficult. Many classifiers simply learn to classify every example as negative, which achieves an accuracy of $99 \%$ but is not at all useful. In the case of decision trees and rule induction, this imbalance is also counterproductive. In addition, some corpora have more examples than the maximum affordable by the learning algorithm, given our computational resources. In this case, it is necessary to reduce the number of examples.

In order to reduce the amount of negative examples, a data selection process similar to clustering is run using the positive examples as the centroids. We define the distance between two examples as the number of features with different values. A negative example is then discarded if the distance to all the positive examples is always greater than a threshold, $D$. The value of $D$ is empirically chosen depending on the corpora and the computational resources available.

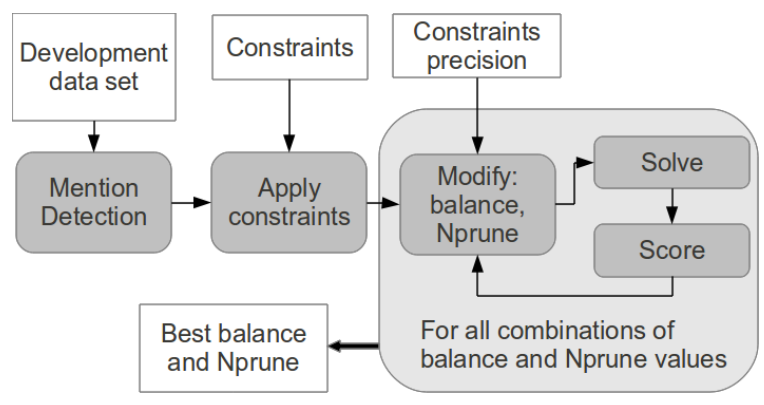

Figure 17

RELAXCOR development process. 
4.3.2 Learning Constraints. Constraints are automatically generated by learning a decision tree and then extracting rules from its leaves using C4.5 software (Quinlan 1993). The algorithm generates a set of rules for each path from the learned tree, then checks whether the rules can be generalized by dropping conditions. These rules become our set of constraints. Other studies have successfully used similar processes to extract rules from a decision tree that are useful in constraint satisfaction algorithms (Màrquez, Padró, and Rodríguez 2000).

The weight assigned to a constraint $\left(\lambda_{k}\right)$ is its precision over the training data $\left(P_{k}\right)$, but shifted by a balance value:

$$
\lambda_{k}=P_{k}-\text { balance }
$$

The precision here refers to the positive class, that is, the ratio between the number of positive examples and the number of examples where the constraint applies. Note that the data selection process (Section 4.3.1) discards some negative examples to learn the constraints, but the weight of the constraints is calculated with the precision of the constraint over the whole training data.

The balance parameter adjusts the constraint weights to improve the balance between precision and recall. On the one hand, a high balance value causes most of the constraints to have a negative weight, with only the most precise having a positive weight. In this case, the system is precise but the recall is low, given that many relations are not detected. On the other hand, a low value for balance causes many lowprecision constraints to have a positive weight, which increases recall but also decreases precision (see Figure 18). The correct value for balance is thus a compromise solution found in the development process, optimizing performance for a specific evaluation measure.

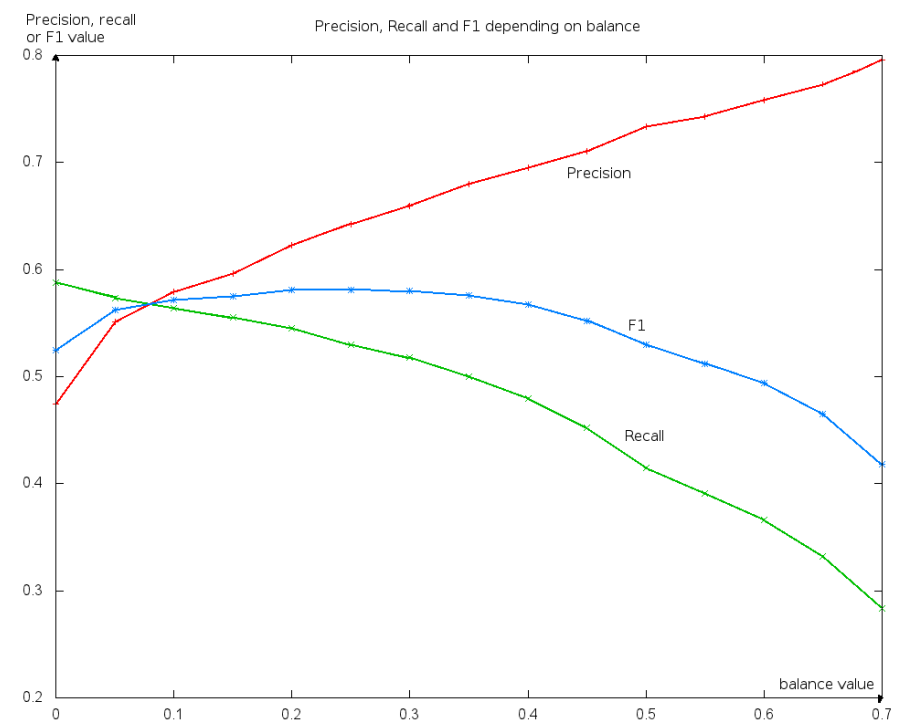

Figure 18

The figure shows MUC's precision (red), recall (green), and $\mathrm{F}_{1}$ (blue) for each balance value in this experiment. Corpus: ACE-2002. 
4.3.3 Pruning. As explained in Section 3.2, when a constraint applies to a set of mentions, a corresponding hyperedge is added to the hypergraph. In the case of the mentionpair model with automatically learned constraints, the most typical case is that each pair of mentions satisfy at least one constraint, which produces an edge for each pair of mentions. There are three main issues to take into account when the problem is represented by an all-connected graph:

- $\quad$ The weight of an edge depends on the weights assigned to the constraints according to Equation (1). Note that the calculation of edge weights is independent of the graph adjacency. This implies that the larger the number of adjacencies, the smaller the influence of a constraint. Consequently, resolution has different results for large and small documents.

- $\quad$ Regarding the second issue, it is notable that some kinds of mention pairs are very weakly informative-for example, pairs such as (pronoun, pronoun). Many stories or discourses have a few main characters (entities) that monopolize the pronouns in the document. This produces many positive training examples for pairs of pronouns matching in gender and person, which may lead the algorithm to produce large coreferential chains joining all these mentions, even for stories where there are many different characters.

- $\quad$ Finally, the computational cost of solving an all-connected graph by relaxation labeling is $O\left(n^{3}\right)$. This cost is easily deduced by examining the algorithm in Figure 13. First, there is a loop for each variable $v_{i}$, and the number of variables is the number of mentions: $n$. Inside this, there is another loop for each label $l$ of $v_{i}$, and the number of labels for $v_{i}$ is $L_{i}=i$. The cost for these two loops is $O\left(\frac{n^{2}}{2}\right)$. Inside the second loop, the support is calculated. The calculation of the support $S_{i l}$ for a vertex $v_{i}$ and label $l$ is an iteration over the incident edges $E\left(v_{i}\right)$, which is equal to $n$ in an all-connected graph. Thus, the adjacency of the vertices depends on the size of the document. Therefore, the final computation cost of the algorithm is $O\left(\frac{n^{3}}{2}\right)$, or $O\left(n^{3}\right)$ taking out the constant value.

The pruning process turns $E\left(v_{i}\right)$ into a constant value $N_{\text {prune }}$. For each vertex's incidence list $E\left(v_{i}\right)$, only a maximum of $N_{\text {prune }}$ edges remain and the others are pruned. In particular, the process keeps the $N_{\text {prune }} / 2$ edges with the largest positive weight and the $N_{\text {prune }} / 2$ with the largest negative weight. The value of $N_{\text {prune }}$ is chosen empirically by maximizing performance over the development data. After pruning, (i) the contribution of the edge weights does not depend on the size of the document; (ii) most edges of the less informative pairs are discarded, avoiding further confusion without limitation on distance or other restrictions that cause a loss of recall; and (iii) computational costs are reduced from $O\left(n^{3}\right)$ to $O\left(n^{2}\right)$, given that the innermost loop has a constant number of iterations $\left(N_{\text {prune }}\right)$.

4.3.4 Reordering. Usually, the vertices of the graph would be placed in the same order as the mentions are found in the document (chronological order). In this manner, $v_{i}$ corresponds to $m_{i}$. As suggested by Luo (2007), however, there is no need to generate the model following that order. In our approach, the first variables have a lower number 
of possible labels. Moreover, an error in the first variables has more influence on the performance than an error in later ones. It is reasonable to expect that placing named entities at the beginning is helpful for the algorithm, given that named entities are usually the most informative mentions.

Reordering only affects the number of possible labels of the variables. The chronological order of the document is taken into account by the constraints, regardless of the graph representation. Our experiments (Sapena, Padró, and Turmo 2010a) confirm that placing named entity mentions first, then nominal mentions, and finally the pronouns, increases the precision considerably. Inside each of these groups, the order is the same as in the document.

\subsection{Training and Development for the Entity-Mention Model}

The training process for the entity-mention model is, in theory, exactly the same as for the mention-pair model, but with predefined influence rules and groups of $N$ mentions instead of pairs. For each combination of influence rule and $N$, the training process has the same steps as explained in previous sections: Learn constraints, apply them to the training data, calculate the weights, and perform the development process to find the optimal balance value. The positive examples are those that satisfy the final condition of the influence rule, and the rest are negative examples. A machine-learning process to discover group constraints has a considerable cost, however, if all the training data need to be evaluated. The number of combinations increases exponentially as the number of implied mentions increases. Moreover, the ratio of positive to negative examples is extremely low, and a data selection process like the one used for pair constraints (Section 4.3.1) has a high computational cost.

For these reasons, the group constraints of our experiments are obtained using only the examples that the mention-pair model could not solve. Thus, after training and running RELAXCOR over an annotated data set using just pair constraints, its errors are now used as examples for training the entity-mention model. The type of errors are those in which three mentions $(N=3)$ corefer $(0,1,2)_{A}$, but the mentionpair model has determined that just two of them corefer and discarded the third one (for example: $\left.(0,1)_{A},(2)_{B}\right)$. Each time an error like this is found, the three mentions correspond to a positive example (corefer) and all other combinations of three mentions between mentions 0 and 2 are considered negative examples. The influence rules for the constraints learned this way are $(0,1)_{A} \Rightarrow(2)_{A},(0,2)_{A} \Rightarrow(1)_{A}$, and $(1,2)_{A} \Rightarrow$ $(0)_{A}$, depending on which mention was wrongly classified by the mention-pair model.

Note that when an entity-mention model has been trained this way, the resolution system is executed using both the mention-pair and entity-mention models at the same time.

Alternatively, constraints for the entity-mention model can be added manually by writing them. Figure 19 shows an example of a manually written entity-mention constraint (i.e., a group constraint with an influence rule). This kind of constraint has great potential to take advantage of the structure of discourses. The example shows how the algorithm can benefit from knowing that nested mentions have some kind of relation. In the case that two coreferring mentions are related with two other mentions with the potential to corefer, the entity-mention model can use this information to find more coreference relations.

The rest of the training and development process is conducted in the same way as for the mention-pair model. The weights of group constraints are obtained by 
The Technical University of Catalonia, sometimes called UPC-Barcelona Tech, is the largest engineering university in Catalonia, Spain. The objectives of the UPC are based on internationalization, as it is [[Spain $]_{0}^{\prime}$ 's technical university with the highest number of international PhD students $]_{1}$ and $\left[[S \text { pain }]_{2}\right.$ 's university with the highest number of international master's degree students $]_{3} \ldots$

Constraint: PN_STR(0,2) \& HEAD_MATCH(1,3) \& NESTED(0,1) \& NESTED(2,3)

Influence rule: $(0,2)_{A},(1)_{B} \Rightarrow(3)_{B}$

\section{Figure 19}

Example of a manually written group constraint using an influence rule to take advantage of the entity-mention model. The constraint expects four mentions where: two of them are proper names and match in their complete strings, the other two match in their heads, mention 0 is inside mention 1 , and mention 2 is inside mention 3 . The influence rule says that when mentions 0 and 2 belong to the same entity (A) but mention 1 belongs to another one (B), then mention 3 should belong to entity B in order to corefer with mention 1. (Text source: Wikipedia. Annotations were manually done for this example.)

evaluating their precision over the training data, and the balance value is determined by a development process. In our experiments, however, the number of group constraints is typically lower than the number of pairwise ones, so there is no need for pruning.

\subsection{Related Work}

In Section 2, we introduced an overview of many approaches, with their classification models and resolution processes (see Figure 5). Our approach can be classified similarly as a one-step resolution that uses the entity-mention model for classification and conducts hypergraph partitioning for the linking process. This classification matches that of the COPA system described in Cai and Strube (2010). Both approaches represent the problem in a hypergraph, where each mention is a vertex, and use hypergraph partitioning in order to find the entities. The differences between these two approaches are substantial, however. The most significant differences are as follows:

- $\quad$ Hypergraph generation. RELAXCOR adds hyperedges to the hypergraph for each group of mentions that satisfy a constraint, whereas COPA adds a hyperedge for each group of mentions that satisfy a feature. Note that the addition of hyperedge weights representing features cannot take advantage of the nonlinear combinations offered by constraints. Actually, in order to incorporate some nonlinearity, COPA needs combined features to introduce information such as mention type (pronoun, proper name, etc.) or distances.

- Resolution algorithm. RELAXCOR uses relaxation labeling in order to satisfy as many constraints as possible. In fact, the hypergraph is just a representation of the problem. COPA uses recursive 2-way partitioning, a hypergraph partitioning algorithm. COPA's main contribution is not the resolution algorithm, but the hypergraph representation of the problem.

- Computational costs. RELAXCOR needs to train a decision tree in order to extract a set of rules to use them as soft constraints. These constraints are then applied to the training data to calculate their weight. COPA does not 
use constraints, which reduces the computational cost of the training process. On the other hand, the cost of the resolution algorithm is $O\left(n^{3}\right)$ for COPA whereas it is $O\left(n^{2}\right)$ in RELAXCOR thanks to the pruning process.

\section{Experiments and Results}

Several experiments have been performed on coreference resolution in order to test our approach. This section includes a short explanation and result analysis of the most significant experiments. First, there is an explanation of a set of experiments to evaluate the performance of coreference resolution and mention detection. The scores are compared with the state of the art in diverse corpora, measures, and languages. Next, our participation in Semeval-2010 and CoNLL-2011 shared tasks is explained in detail with performance, comparisons, and error analysis. Finally, a set of experiments using the entity-mention model are described.

The framework used in our experiments consists of widely used corpora and measures to facilitate replication and comparison. Corpora used are ACE 2002 (NIST 2003), the same portion of OntoNotes v2.0 used in Semeval-2010 (Recasens et al. 2010), and the same portion of OntoNotes v4.0 used in CoNLL Shared Task 2011 (Pradhan et al. 2011). Regarding the measures, we used MUC (Vilain et al. 1995), $B^{3}$ (Bagga and Baldwin 1998), and two variants of CEAF (Luo 2005): mention-based (CEAFm) and entity-based (CEAFe).

\subsection{Mention Detection}

The performance of the mention detection system achieves a good recall, higher than $90 \%$, but a low precision, as published in Sapena, Padró, and Turmo (2011) and reproduced in Table 1. The OntoNotes corpora have been used for this experiment, as they were used in CoNLL-2011. Given that the mention detection in a pipeline combination acts as a filter, recall should be kept high, as a loss of recall at the beginning would result in a loss of performance in the rest of the process. At this point, however, the precision is not a priority as long as it remains reasonable, given that the coreference resolution process is able to determine that many mentions are singletons. Moreover, the evaluation of precision on the OntoNotes corpora only take into account mentions included in a coreference chain, not singletons. The RELAXCOR output, however, includes singletons. This means that the precision value is not really evaluating the precision of the mention detection system. A fair evaluation of mention detection should be performed in a corpus with annotations of every referring expression, but such a corpus is not available as far as we know.

The most typical error made by the system is to include extracted NPs that are not referential (e.g., predicative and appositive phrases) and mentions with incorrect boundaries. The incorrect boundaries are mainly due to errors in the predicted syntactic

Table 1

Mention detection results on OntoNotes (Corpus: CoNLL-2011 Shared Task).

\begin{tabular}{lccc}
\hline OntoNotes & Recall & Precision & $\mathrm{F}_{1}$ \\
\hline Development & 92.45 & 27.34 & 42.20 \\
Test & 92.39 & 28.19 & 43.20
\end{tabular}


Table 2

Results on ACE-phase02.

\begin{tabular}{lccc|c|cccc}
\hline & bnews & npaper & nwire & \multicolumn{5}{|c}{ Global } \\
\hline Metric: & & CEAF & & CEAF & \multicolumn{3}{|c}{$B^{3}$} \\
\hline Model & F1 & F1 & F1 & F1 & P & R & F1 \\
\hline RELAXCOR & $\mathbf{6 9 . 5}$ & $\mathbf{6 7 . 3}$ & $\mathbf{7 2 . 1}$ & $\mathbf{6 9 . 7}$ & 85.3 & 66.8 & 74.9 \\
MaxEnt+ILP (Denis 2007) & - & - & - & 66.2 & 81.4 & 65.6 & 72.7 \\
Rankers (Denis 2007) & 65.7 & 65.3 & 68.1 & 67.0 & 79.8 & 66.8 & 72.7
\end{tabular}

column and some mention annotation discrepancies. Furthermore, the coreference annotation of OntoNotes used in CoNLL-2011 included verbs as anaphors of some verbal nominalizations. But verbs are not detected by our mention detection system, so most of the missing mentions are verbs. The methodology of the mention detection system is explained in Section 4.1.

\subsection{State-of-the-Art Comparison}

RELAXCOR performance has been compared several times with other published results from state-of-the-art systems. We claimed Sapena, Padró, and Turmo (2010a) to have the best performance for the ACE-phase 02 corpus, using true mentions in the input and evaluating with the CEAF and $B^{3}$ measures. The table comparing scores with the best results found at that moment is reproduced as Table 2 .

The approach is also compared with the state of the art in two competitions: SemEval-2010 (Sapena, Padró, and Turmo 2010b) and CoNLL-2011 (Sapena, Padró, and Turmo 2011). RELAXCOR achieved one of the best performances in SemEval-2010, but contradictory results across measures prevented the organization from determining a

\begin{tabular}{|l|c|c|c|c|c|c|c|}
\hline System & $\mathrm{MD}$ & $\mathrm{MUC}$ & $\mathrm{B}-\mathrm{CUBED}$ & $\mathrm{CEAF}_{m}$ & $\mathrm{CEAF}_{e}$ & BLANC & $\begin{array}{c}\text { Official } \\
\end{array}$ \\
$\mathrm{F}$ & $\mathrm{F}^{1}$ & $\mathrm{~F}^{2}$ & $\mathrm{~F}$ & $\mathrm{~F}^{3}$ & $\mathrm{~F}$ & $\frac{F^{1}+F^{2}+F^{3}}{3}$ \\
\hline lee & 70.70 & 59.57 & 68.31 & 56.37 & 45.48 & 73.02 & 57.79 \\
sapena & 43.20 & 59.55 & 67.09 & 53.51 & 41.32 & 71.10 & 55.99 \\
chang & 64.28 & 57.15 & 68.79 & 54.40 & 41.94 & 73.71 & 55.96 \\
nugues & 68.96 & 58.61 & 65.46 & 51.45 & 39.52 & 71.11 & 54.53 \\
santos & 65.45 & 56.65 & 65.66 & 49.54 & 37.91 & 69.46 & 53.41 \\
song & 67.26 & 59.95 & 63.23 & 46.29 & 35.96 & 61.47 & 53.05 \\
stoyanov & 67.78 & 58.43 & 61.44 & 46.08 & 35.28 & 60.28 & 51.92 \\
sobha & 64.23 & 50.48 & 64.00 & 49.48 & 41.23 & 63.28 & 51.90 \\
kobdani & 61.03 & 53.49 & 65.25 & 42.70 & 33.79 & 62.61 & 51.04 \\
zhou & 62.31 & 48.96 & 64.07 & 47.53 & 39.74 & 64.72 & 50.92 \\
charton & 64.30 & 52.45 & 62.10 & 46.22 & 36.54 & 64.20 & 50.36 \\
yang & 63.93 & 52.31 & 62.32 & 46.55 & 35.33 & 64.63 & 49.99 \\
hao & 64.30 & 54.47 & 61.01 & 45.07 & 32.67 & 65.35 & 49.38 \\
xinxin & 61.92 & 46.62 & 61.93 & 44.75 & 36.23 & 64.27 & 48.46 \\
zhang & 61.13 & 47.28 & 61.14 & 44.46 & 35.19 & 65.21 & 48.07 \\
kummerfeld & 62.72 & 42.70 & 60.29 & 45.35 & 38.32 & 59.91 & 47.10 \\
zhekova & 48.29 & 24.08 & 61.46 & 40.43 & 35.75 & 53.77 & 40.43 \\
irwin & 26.67 & 19.98 & 50.46 & 31.68 & 25.21 & 51.12 & 31.28 \\
\hline
\end{tabular}

Figure 20

RELAXCOR (sapena) achieved the second position in the official closed track (CoNLL-2011). 
Table 3

Results of RELAXCOR on development data (SemEval-2010).

\begin{tabular}{lccc|ccc|ccc}
\hline & \multicolumn{3}{c|}{ CEAF } & \multicolumn{3}{c|}{ MUC } & \multicolumn{3}{c}{$\mathrm{B}^{3}$} \\
\hline language & $\mathrm{R}$ & $\mathrm{P}$ & $\mathrm{F}_{1}$ & $\mathrm{R}$ & $\mathrm{P}$ & $\mathrm{F}_{1}$ & $\mathrm{R}$ & $\mathrm{P}$ & $\mathrm{F}_{1}$ \\
\hline ca & 69.7 & 69.7 & 69.7 & 27.4 & 77.9 & 40.6 & 67.9 & 96.1 & 79.6 \\
es & 70.8 & 70.8 & 70.8 & 30.3 & 76.2 & 43.4 & 68.9 & 95.0 & 79.8 \\
en-closed & 74.8 & 74.8 & 74.8 & 21.4 & 67.8 & 32.6 & 74.1 & 96.0 & 83.7 \\
en-open & 75.0 & 75.0 & 75.0 & 22.0 & 66.6 & 33.0 & 74.2 & 95.9 & 83.7
\end{tabular}

winner. In addition, RELAXCOR achieved second position in the CoNLL-2011 Shared Task; Figure 20 reproduces the official table of results. Following sections describe the shared tasks in detail.

Finally, the performance of RELAXCOR is again compared with two other state-ofthe-art systems in Màrquez, Recasens, and Sapena (2012).

5.2.1 SemEval-2010. The goal of SemEval-2010 task 1 (Recasens et al. 2010) was to evaluate and compare automatic coreference resolution systems for six different languages in four evaluation settings and using four different evaluation measures. This complex scenario aimed at providing insight into several aspects of coreference resolution, including portability across languages, relevance of linguistic information at different levels, and behavior of alternative scoring measures. The task attracted considerable attention from a number of researchers, but only six teams submitted results. Moreover, participating systems did not run their systems for all the languages and evaluation settings, thus making direct comparisons among all the involved dimensions very difficult.

RELAXCOR participated in the SemEval task for English, Catalan, and Spanish (Sapena, Padró, and Turmo 2010b). At the time, the system was not ready to detect mentions. Thus, participation was restricted to the gold-standard evaluation, which included the manual annotated information and also provided the mention boundaries.

RELAXCOR results for development and test data sets are shown in Tables 3 and 4, respectively. The version of RELAXCOR used in SemEval had a balance value fixed to 0.5 , which proved to be an inadequate value. Thus, the results have high precision but a very low recall. This situation produced high scores with the CEAF and $B^{3}$ measures, due in part to the annotated singletons. The system was penalized by measures based on pair-linkage, however, particularly MUC. Although RELAxCOR had the highest

Table 4

Results of RELAXCOR on test data (SemEval-2010).

\begin{tabular}{|c|c|c|c|c|c|c|c|c|c|c|c|c|}
\hline \multirow{2}{*}{ language } & \multicolumn{3}{|c|}{ CEAF } & \multicolumn{3}{|c|}{ MUC } & \multicolumn{3}{|c|}{$\mathrm{B}^{3}$} & \multicolumn{3}{|c|}{ BLANC } \\
\hline & $\mathrm{R}$ & $\mathrm{P}$ & $\mathrm{F}_{1}$ & $\mathrm{R}$ & $\mathrm{P}$ & $\mathrm{F}_{1}$ & $\mathrm{R}$ & $\mathrm{P}$ & $\mathrm{F}_{1}$ & $\mathrm{R}$ & $\mathrm{P}$ & BLANC \\
\hline \multicolumn{13}{|c|}{ Information: closed Annotation: gold } \\
\hline $\mathrm{ca}$ & 70.5 & 70.5 & 70.5 & 29.3 & 77.3 & 42.5 & 68.6 & 95.8 & 79.9 & 56.0 & 81.8 & 59.7 \\
\hline es & 66.6 & 66.6 & 66.6 & 14.8 & 73.8 & 24.7 & 65.3 & 97.5 & 78.2 & 53.4 & 81.8 & 55.6 \\
\hline en & 75.6 & 75.6 & 75.6 & 21.9 & 72.4 & 33.7 & 74.8 & 97.0 & 84.5 & 57.0 & 83.4 & 61.3 \\
\hline \multicolumn{13}{|c|}{ Information: open Annotation: gold } \\
\hline en & 75.8 & 75.8 & 75.8 & 22.6 & 70.5 & 34.2 & 75.2 & 96.7 & 84.6 & 58.0 & 83.8 & 62.7 \\
\hline
\end{tabular}


precision scores (even with MUC), the recall was low enough to finally obtain low scores for $F_{1}$.

Regarding the test scores of the comparison with other participants (Recasens et al. 2010), RELAXCOR obtained the best performance for Catalan (CEAF and $B^{3}$ ), English (closed: CEAF and $B^{3}$; open: $B^{3}$ ), and Spanish $\left(B^{3}\right)$. Moreover, RELAXCOR was the most precise system under all metrics in all languages, except for CEAF in English-open and Spanish. This confirms the robustness of the results of RELAXCOR, but also highlights the necessity of searching for a balance value other than 0.5 to increase the recall of the system without losing much by way of precision. Indeed, the idea of using development (Section 4.3) to adapt the balance value occurred after these results were obtained.

The incorporation of WordNet to the English run of RELAXCOR was the only difference between our implementation in the English-open and English-closed tasks. The scores were slightly higher when using WordNet, but not significantly so $(75.8 \%$ vs. $75.6 \%$ for CEAF and $34.2 \%$ vs. $33.7 \%$ for MUC). Analyzing the MUC scores, note that the recall improves (from $21.9 \%$ to $22.6 \%$ ), while the precision decreases a little (from $74.4 \%$ to $70.5 \%$ ), which corresponds to the information and noise that WordNet typically provides.

More recent results of RELAXCOR on the same corpora are published in Màrquez, Recasens, and Sapena (2012).

5.2.2 CoNLL-2011. The CoNLL-2011 Shared Task was based on the English portion of the OntoNotes 4.0 data $^{5}$ (Pradhan et al. 2011). As is customary for CoNLL tasks, there was a closed and an open track. For the closed track, systems were limited to using the distributed resources, in order to allow a fair comparison of algorithm performance, whereas the open track allowed for almost unrestricted use of external resources in addition to the provided data. About 65 different groups demonstrated interest in the shared task by registering on the task Web page. Of these, 23 groups submitted system outputs on the test set during the evaluation week. Eighteen groups submitted only closed track results, three groups only open track results, and two groups submitted both closed and open track results.

RELAXCOR participated in the closed track CoNLL task (Sapena, Padró, and Turmo 2011). All the knowledge required by the feature functions was obtained from the annotations of the corpus, and no external resources were used with the exception of WordNet, gender and number information, and sense inventories. All of these were allowed by the task organization and are available on their Web site.

The results obtained by RELAXCOR can be found in Tables 5 and 6 . Due to the lack of annotated singletons, mention-based metrics $B^{3}$ and CEAF produce lower scores (near $60 \%$ and $50 \%$, respectively) than typically achieved with different annotations and mapping policies (usually near $80 \%$ and $70 \%$ ). Moreover, the requirement that systems use automatic preprocessing and do their own mention detection increases the difficulty of the task, which obviously decreases the scores in general. The official ranking score was the arithmetic mean of the F-scores of MUC, $B^{3}$, and CEAFe.

The MUC measure is link-based and does not take singletons into account, anyway. Thus, it is the only one comparable with the state of the art at this point. The results obtained with the MUC scorer show an improvement in RELAXCOR's recall, a feature that needed improvement given the remarkably low SemEval-2010 results with MUC. Note that these improvements in MUC scores, specially in recall, are mainly due to

5 CoNLL-2011 Shared Task Web site: http://conll.bbn. com. 
Table 5

RELAXCOR results on the development data set (CoNLL-2011).

\begin{tabular}{lccc}
\hline Measure & Recall & Precision & $\mathrm{F}_{1}$ \\
\hline Mention detection & 92.45 & 27.34 & 42.20 \\
mention-based CEAF & 55.27 & 55.27 & 55.27 \\
entity-based CEAF & 47.20 & 40.01 & 43.31 \\
MUC & 54.53 & 62.25 & 58.13 \\
$\mathrm{~B}^{3}$ & 63.72 & 73.83 & 68.40 \\
$\left(\mathrm{CEAFe}+\mathrm{MUC}+\mathrm{B}^{3}\right) / 3$ & - & - & 56.61
\end{tabular}

Table 6

RELAXCOR official test results (CoNLL-2011).

\begin{tabular}{lccc}
\hline Measure & Recall & Precision & $\mathrm{F}_{1}$ \\
\hline Mention detection & 92.39 & 28.19 & 43.20 \\
mention-based CEAF & 53.51 & 53.51 & 53.51 \\
entity-based CEAF & 44.75 & 38.38 & 41.32 \\
MUC & 56.32 & 63.16 & 59.55 \\
B $^{3}$ & 62.16 & 72.08 & 67.09 \\
BLANC & 69.50 & 73.07 & 71.10 \\
$\left(\mathrm{CEAFe}+\mathrm{MUC}+\mathrm{B}^{3}\right) / 3$ & - & - & $\mathbf{5 5 . 9 9}$
\end{tabular}

the introduction of the balance value in the development process but also to many other refinements done in the whole process such as new feature functions and bug fixing.

RELAXCOR achieved second position in the official closed track results, as shown in Figure 20. The final column shows the official ranking score. The difference from the system in first place is 1.8 points, which is statistically significant, whereas the difference to third position is just 0.03 points and is not significant. The winning system-Stanford (Lee et al. 2011)—does not use machine learning but combines many heuristics to join mentions and partial entities, starting with the most precise ones. It is thought that the difference between RELAXCOR and Stanford's system is mainly due to their use of sophisticated handwritten heuristics instead of our automatically learned constraints. Note that Lee et al. (2011) solve coreferences by applying first the most precise constraints. RELAXCOR also solves first the most precise constraints given that these ones have the highest weights and are the most influencing ones.

\subsection{Languages}

Sapena, Padró, and Turmo (2010b), and Màrquez, Recasens, and Sapena (2012) show the performance of our approach for English, Catalan, and Spanish. The scores for Spanish and Catalan do not seem as good as for English, because the system was originally designed with the English language in mind. As a result, it does not include languagespecific features for Spanish and Catalan, such as whether a mention is an elliptical subject or not. Despite this, RELAXCOR scores for Catalan and Spanish are the best among the state of the art. 
Table 7

Comparison of RELAXCOR results using just the mention-pair model $(N=2)$ with those also using the entity-mention model $(N=3)$ (Corpus: SemEval-2010).

\begin{tabular}{llccc}
\hline & Measure & Precision & Recall & \multicolumn{1}{c}{$\mathrm{F}_{1}$} \\
\hline & CEAFm & 81.73 & 81.73 & 81.73 \\
$\|$ & MUC & 72.92 & 54.17 & 62.17 \\
$Z$ & $B^{3}$ & 91.87 & 82.87 & 87.14 \\
& CEAFe & 81.95 & 90.47 & 86.00 \\
$\infty$ & CEAFm & 82.02 & 82.02 & 82.02 \\
$\|$ & MUC & 73.01 & 54.28 & 62.27 \\
$Z$ & $B^{3}$ & 91.59 & 83.12 & 87.15 \\
& CEAFe & 82.10 & 90.63 & 86.15
\end{tabular}

\subsection{Experiments with the Entity-Mention Model}

Constraints for the entity-mention model are automatically obtained using the training data examples that the mention-pair model could not solve, with predefined influence rules and limited to $N=3$. The training process is explained in Section 4.4. Experiments with the entity-mention model are conducted using both models at the same time. The goal of the experiments is to improve the performance of the mention-pair model itself.

Table 7 shows the experimental results using the SemEval-2010 English corpus. The table compares the entity-mention results (RELAXCOR using $N=3$ constraints with influence rules, including the whole set of $N=2$ constraints) with those using mentionpairs (RELAXCOR using just $N=2$ constraints). The entity-mention model outperforms the mention-pair model. The number of really useful examples (i.e., mentions wrongly classified by the mention-pair model but correctly classified by the entity-mention model), however, is low. Consequently, the difference in their scores is not significant. The $N=3$ constraints have a good precision and also an acceptable recall, although most of the mentions affected by these constraints were already affected and correctly solved by the mention-pair model. Further research is needed in order to find more useful constraints, either by writing more elaborate group constraints or finding a better system that automatically finds them.

These results may be somewhat justified, because the entity-mention model uses the same feature functions and, consequently, the same information as the mentionpair model. In fact, only the new information is that information already included in the conditions of the influence rules, which take into account the entities assigned to each mention during resolution. In addition, group constraints can also include, in an implicit way, information about the structure of the discourse. It seems clear, however, that this new information is either minimal or not relevant enough. Figure 21 shows an example of a learned entity-mention constraint.

Constraint: GENDER_NO_(0,1) $=0$ \& STR_MATCH(0,1) $=0$ \& ORGANIZA-
TION(0)=0 \& POSSESSIVE(1) \& NUMBER_NO(1,2) = 0 \& NUMBER_UN(1,2)
$=0$ \& DIST_SEN_L3(0,1) \& DIST_SEN_L3(0,2)
Influence rule: $(0,2)_{A} \Rightarrow(1)_{A}$

\section{Figure 21}

Example of a learned $N=3$ constraint. 
Even though the obtained performance does not significantly outperform the mention-pair model, we can draw some positive conclusions from these experiments. First of all, the approach is ready to use either model (mention-pair or entity-mention) in a constructive way. As soon as new feature functions specific to entity-mention models appear, the results will reflect this. One research line to follow in this field is the incorporation of feature functions following discourse theories, such as focusing and centering. Another research line is the introduction of world knowledge using these models, as explained in the next section.

\section{Adding World Knowledge to Coreference Resolution}

Often, common sense and world knowledge is essential to resolve coreferences. For example, we can find coreferential mentions in any newspaper, such as \{Obama, USA President $\},\{$ Messi, Barcelona striker $\}$, or $\{$ Beirut, the Lebanese capital $\}$.

In order to know the importance of the coreference links that are missed due to a lack of world knowledge, the partial and total scores of RELAXCOR on the test data set of OntoNotes 2.0 (the same data set used for the English task in SemEval-2010) are shown in Table 9 for each mention class described in Table 8. Analyzing the table, we observe that PN_N, CN_P, and CN_N are the classes with the lowest recall, especially PN_N and $C N_{-} N$. In addition, PN_N and $C_{-} N_{-N}$ have the lowest precision. The final column shows the number of mentions corresponding to the class of that row and the percentage representing the total number of coreferent mentions. Note that these three classes together represent $27 \%$ of coreferent mentions.

According to Màrquez, Recasens, and Sapena (2012), Stoyanov et al. (2009), and Pradhan et al. (2007), these results can be roughly generalized to any other system using similar information, and even other languages. Therefore, these classes require attention in order to improve global performance, and the fact that lexical, morphological, syntactic, and semantic levels are not very useful to deal with them encourages the research on adding world knowledge to coreference resolution systems. In stateof-the-art systems, we can find some attempts to add world knowledge to coreference resolution, using Wikipedia (Ponzetto and Strube 2006; Uryupina et al. 2011) or YAGO and Freenet (Rahman and Ng 2011a).

Table 8

Description of the mention classes for English documents.

\begin{tabular}{|c|c|}
\hline Class & Description \\
\hline PN_E & $\begin{array}{l}\text { NPs headed by a Proper Name that Exactly match (excluding case and the } \\
\text { determiner) at least one preceding mention in the same coreference chain. }\end{array}$ \\
\hline PN_P & $\begin{array}{l}\text { NPs headed by a Proper Name that Partially match (i.e., head match or overlap, } \\
\text { excluding case) at least one preceding mention in the same coreference chain. }\end{array}$ \\
\hline PN_N & $\begin{array}{l}\text { NPs headed by a Proper Name that do Not match any preceding mention in } \\
\text { the same coreference chain. }\end{array}$ \\
\hline $\begin{array}{l}\text { CN_E } \\
\text { CN_P } \\
\text { CN_N }\end{array}$ & $\begin{array}{l}\text { Same definitions as in PN_E, PN_P, and PN_N, } \\
\text { but referring to NPs headed by a Common Noun. }\end{array}$ \\
\hline $\begin{array}{l}P \_1 \cup 2 \\
P \_3 G \\
P \_3 U\end{array}$ & $\begin{array}{l}\text { First- and second-person pronouns that corefer with a preceding mention. } \\
\text { Gendered third-person pronouns that corefer with a preceding mention. } \\
\text { Ungendered third-person pronouns that corefer with a preceding mention. }\end{array}$ \\
\hline
\end{tabular}


Table 9

Results of RELAXCOR in English OntoNotes from SemEval-2010 without world knowledge.

\begin{tabular}{|c|c|c|c|c|c|}
\hline measure & class & Pre & $\operatorname{Rec}$ & F1 & quantity \\
\hline \multirow{9}{*}{ MUC } & PN_E & 99.7 & 99.4 & 99.6 & $356(18 \%)$ \\
\hline & PN_P & 94.5 & 77.9 & 85.4 & $222(12 \%)$ \\
\hline & PN_N & 5.3 & 1.3 & 2.1 & $75(04 \%)$ \\
\hline & CN_E & 97.3 & 71.8 & 82.6 & $149(08 \%)$ \\
\hline & CN_P & 87.3 & 36.0 & 51.0 & $172(09 \%)$ \\
\hline & CN_N & 22.6 & 2.5 & 4.5 & $278(14 \%)$ \\
\hline & $P_{-} 1 \cup 2$ & 74.5 & 61.2 & 67.2 & $134(07 \%)$ \\
\hline & P_3G & 88.8 & 85.0 & 86.9 & $187(10 \%)$ \\
\hline & P_3U & 78.1 & 59.3 & 67.4 & $356(18 \%)$ \\
\hline MUC & & 74.4 & 59.9 & 66.4 & \\
\hline CEAFm & & 83.0 & 83.0 & 83.0 & \\
\hline$B^{3}$ & & 91.8 & 84.6 & 88.1 & \\
\hline
\end{tabular}

This section presents our approach to incorporating world knowledge to coreference resolution, represented in Figure 22. The nature of our model allows the integration of world knowledge encoded not only as features, but also as constraints, which is a more expressive and natural way.

The work presented in this section is intended as a proof-of-concept for the ability of RELAXCOR to absorb knowledge from heterogeneous sources. Results show that although the algorithm is able to successfully handle the added knowledge, the performance is hardly increased due to the noisy nature of the knowledge automatically extracted from Wikipedia.

The approach proceeds in two phases. First, given a document, the world knowledge potentially useful for the resolution of coreferences is acquired from Wikipedia, and second, this knowledge is incorporated to RELAXCOR using two alternative models: feature functions and constraints. These phases are described in Sections 6.1 and 6.2, respectively. Finally, Section 6.3 describes our experiments and analyzes their results.

\subsection{Acquiring World Knowledge}

Our methodology to acquire world knowledge useful for coreference resolution consists of finding the real-world entities occurring in the document (i.e., Entity Linking) and extracting information related to them from Wikipedia.

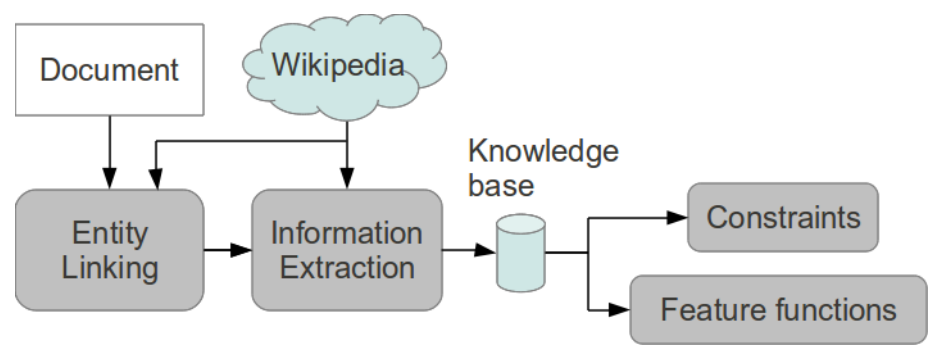

Figure 22

Process to add information from Wikipedia to coreference resolution. 
6.1.1 Entity Linking. One approach to finding real-world entities mentioned in a document is to select the NE mentions of that document and to disambiguate them in order to determine which entities in the real world-in our case, which Wikipedia entriesare referred to by the mentions. Using every NE mention in a document, however, may add noise to the process of coreference resolution. For instance, consider a document with Bill Clinton and, some sentences later, Clinton. If we try to get information about Clinton from Wikipedia, we obtain a page about the English family name Clinton with a lot of non-relevant information that may lead to erroneous results. Given that Bill Clinton appears in the same document, it seems more convenient to select the most informative NE mention and discard the less informative ones, like Clinton, which are probably pointing to the same real-world entity. This is why we just take into account the most informative NE mentions, called MI mentions from now on.

We obtain MI mentions as follows: We build cliques formed by groups of mentions where the ALIAS function is true for all the pairs, and all mentions in the group belong to the same class (Person, Organization, or Location). Finally, the longest NE mention from each group is selected as an MI mention.

After this selection process, each MI mention is disambiguated by using Google as an information retrieval system to find the most relevant pages in Wikipedia. The query is generated from the MI mention as the mention head plus all nouns, proper names, and adjectives that appear immediately before it. From the results provided by Google, we select as the real world entity for the MI mention the first URL that corresponds to a Wikipedia entry and includes the head of the MI mention (or a string that matches as an alias) in the title or in the first sentence of the first paragraph. ${ }^{6}$ If no result is found, we assume that the MI mention does not exist in Wikipedia.

6.1.2 Information Extraction. For each Wikipedia entry obtained in the entity disambiguation step, we extract information from the description, the infobox, and the categories of the entry, and also from other Wikipedia pages linking to that entry, as found in the "What Links Here" section. Concretely, we extract all names (i.e., official names, nicknames, and aliases), as well as properties indicating the most descriptive aspects or qualities of the entity.

The first paragraph of a Wikipedia entry is considered the description of the entry. The description typically starts with the complete name of the entity, some aliases, and the most descriptive properties of the entity. After preprocessing the text, the first NE is extracted as the official name. Next, a set of patterns are used to extract aliases (e.g., "sometimes called <alias $>$ ") or properties (e.g., "<mention> be/become NP-<property>"). ${ }^{7}$

From the infobox, all the contents of the following fields are extracted: fullname, name, office, title, profession, company_name, playername, occupation, nickname, official_name, native_name, settlement_type, type. In addition, all categories associated with the entry are also extracted as properties.

Finally, from each page that links to the current entry following the "What Links Here" section, those sentences including the link are selected. From each one of them, the anchor text used to link the entry is extracted as a name. In addition, the following

6 We also discard special Wikipedia pages, such as disambiguation pages or pages with names that do not contain the character ":".

7 We extract as properties both the NP (e.g., "American politician") and its head (e.g., "politician") in order to get more general properties as well. 
expressions are extracted as properties using patterns: the set of nouns and adjectives to the left of the anchor text, the set of NPs in apposition to the link, and the set of NPs denoting a property of a list of entries in which one of them is the current one (e.g., " $<\mathrm{NP}>-<$ property $>$ such as entry 1 , entry $2, \ldots$, entry" , $_{n}$.

We take the frequency of the extracted expression as the confidence value associated with each expression-the most repeated expressions are the most reliable. In order to avoid incorrect information as much as possible, we define a threshold below which all the extracted names and properties are discarded.

\subsection{Incorporating World Knowledge to the Models}

Two approaches for the incorporation of the knowledge extracted from Wikipedia have been studied. The first is to add some feature functions for the mention-pair model that evaluate whether a pair of mentions may corefer according to Wikipedia's information, similar to other state-of-the-art studies (Ponzetto and Strube 2006; Rahman and Ng 2011a). The second approach adds a set of constraints to the hypergraph connecting groups of mentions, using the entity-mention model.

6.2.1 Feature Functions. In this approach, new feature functions are added to evaluate pairs of mentions, and some learned constraints may use them as any other feature function. These feature functions are only applied to pairs $\langle M I, X\rangle$, where $M I$ is a MI mention and $X$ is any other mention but a pronoun, and use the information extracted from Wikipedia to determine their value. Concretely, the feature functions used in our experiments are the following ones:

- WIKI_ALIAS $(M I, X)$ : returns true if the head of $X$ is another MI mention of the same entry as $M I$, or $X$ matches one of the names extracted from Wikipedia for $M I$.

- WIKI DESC $(M I, X)$ : returns true if all the $X$ terms are included in one of the properties extracted from Wikipedia for $M I$.

6.2.2 Constraints. In this approach, world knowledge is incorporated by adding constraints relating the mentions that may corefer given the extracted information about the entities. In this case, the features of the previous model are now replaced by constraints. In addition, other constraints can be added to take advantage of the entity-mention model. The following is a list of constraints used in our experiments:

- Constraint cAlias is added for each pair of mentions that satisfy the same conditions as WIKI_ALIAS.

- Constraint $c$ Desc is added for each pair of mentions that satisfy the same conditions as WIKI DESC.

- Constraint cWiki3, a $N=3$ constraint, is added for each combination of three mentions $(0,1,2)$ where 0 is a MI mention, 1 is a NE mention alias of 0 , and 2 is a nominal mention or a NE mention that satisfies WIKI_ALIAS or WIKI_DESC with 0 . This constraint tries to link the nominal mention with the closest NE mention that may corefer. The influence rule is $(0,1)_{A} \Rightarrow(2)_{A}$, that is, 2 is influenced when 0 and 1 corefer. 
- $\quad$ Constraint cStructWiki3, an $N=3$ constraint, is added for each combination of three mentions $(0,1,2)$ where 0 is an MI mention, 1 is an NP that satisfies WIKI_ALIAS or WIKI_DESC with 0 , and 2 is an NE mention alias of 0 . In addition, the three mentions have the same syntactic function and are found in consecutive sentences. The influence rule associated with this constraint is $(0,2)_{A} \Rightarrow(1)_{A}$, that is, 1 is influenced when 0 and 2 corefer.

Figure 23 shows examples of the constraints cWiki3 and cStructWiki3. The idea behind cWiki3 is to link the nominal mention (2, The organization) with a closer mention in the document than the MI mention (0, the Organization of Petroleum Exporting Countries). Linking nearest mentions may take advantage of information given by other constraints, such as syntactic patterns. When the Organization of Petroleum Exporting Countries is tending to corefer with OPEC, mention The organization is influenced by both mentions. The second case, cStructWiki3, takes advantage of a typical discourse structure where the same entity is the subject of some consecutive sentences. First mention 0, Google Inc., is the MI mention, whereas 2 (Google) is just an alias. Between them we find a nominal mention (The company), which we expect to solve using world knowledge. Both $N=3$ constraints are expected to have high precision but low recall.

Note that cAlias and cWiki are equivalent to the feature functions of the previous model. The difference is that, in the case of constraints, they are always applied when WIKI_ALIAS and WIKI_DESC are true, and so their weight is added to the edge weight of that pair in the hypergraph. In the model using feature functions, however, the constraints learned by the model may or may not include those features.

\title{
6.3 Experiments and Results
}

The experiments consist of the execution of RELAXCOR using each one of the models to incorporate information. RELAXCOR + features incorporates the new features to the original model and repeats the training process from the beginning. Constraints are learned using these new feature functions mixed with all the others (a detailed list of features is in Section 4.2). RELAXCOR + constraints incorporates the new constraints. In this case, the learning process uses the constraints already learned for RELAXCOR and adds the new constraints to the model. The training process is then applied normally to compute the weight of the constraints using their precision in the training files.

Table 10 shows the results obtained when adding world knowledge compared with the results of RELAXCOR without world knowledge. The first three columns list the

\author{
cWiki3 \\ The organization is scheduled to meet in Vienna... \\ cStructWiki3 \\ Google Inc. is offering new applications... \\ The company is going to... \\ Predictably, Google has highlighted user profiles...
}

Output from the Organization of Petroleum Exporting Countries is already...

As a result, the effort by some oil ministers to get OPEC to approve...

\section{Figure 23}

Examples of the application of $N=3$ constraints cWiki3 and cStructWiki3. 
Table 10

Results of RELAXCOR on English OntoNotes 2.0 from SemEval-2010 with world knowledge. The baseline is RELAXCOR using mention-pair model, features, is RELAXCOR using features WIKI_ALIAS and WIKI_DESC, and constraints stands for RELAXCOR with the set of constraints in Section 6.2.2. Gains over the baseline are boldfaced and losses are in italics.

\begin{tabular}{ll|rrr|rrr|rrr}
\hline & & \multicolumn{3}{c|}{ baseline } & \multicolumn{3}{c|}{ features } & \multicolumn{3}{c}{ constraints } \\
\hline measure & class & Pre & Rec & \multicolumn{1}{c|}{ F1 } & Pre & Rec & \multicolumn{1}{c}{ F1 } & Pre & Rec & \multicolumn{1}{c}{ F1 } \\
\hline \multirow{4}{*}{} & PN_E & 99.7 & 99.4 & 99.6 & $\mathbf{1 0 0}$ & 98.0 & 99.0 & $\mathbf{1 0 0}$ & 99.2 & 99.6 \\
& PN_P & 94.5 & 77.9 & 85.4 & 92.9 & 76.6 & 84.0 & 93.6 & $\mathbf{7 8 . 8}$ & $\mathbf{8 5 . 6}$ \\
& PN_N & 5.3 & 1.3 & 2.1 & $\mathbf{1 5 . 0}$ & $\mathbf{4 . 0}$ & $\mathbf{6 . 3}$ & $\mathbf{1 4 . 8}$ & $\mathbf{5 . 3}$ & $\mathbf{7 . 8}$ \\
MUC & CN_E & 97.3 & 71.8 & 82.6 & 97.3 & $\mathbf{7 2 . 5}$ & $\mathbf{8 3 . 1}$ & 97.3 & $\mathbf{7 2 . 5}$ & $\mathbf{8 3 . 1}$ \\
& CN_P & 87.3 & 36.0 & 51.0 & $\mathbf{9 0 . 2}$ & $\mathbf{4 3 . 0}$ & $\mathbf{5 8 . 3}$ & $\mathbf{8 9 . 2}$ & $\mathbf{4 3 . 0}$ & $\mathbf{5 8 . 0}$ \\
& CN_N & 22.6 & 2.5 & 4.5 & $\mathbf{3 2 . 1}$ & $\mathbf{3 . 2}$ & $\mathbf{5 . 9}$ & $\mathbf{3 1 . 0}$ & $\mathbf{3 . 2}$ & $\mathbf{5 . 9}$ \\
& P_1U2 & 74.5 & 61.2 & 67.2 & $\mathbf{7 6 . 9}$ & 59.7 & 67.2 & $\mathbf{7 7 . 1}$ & 60.4 & $\mathbf{6 7 . 8}$ \\
& P_3G & 88.8 & 85.0 & 86.9 & 87.6 & $\mathbf{8 6 . 6}$ & $\mathbf{8 7 . 1}$ & 87.6 & $\mathbf{8 6 . 6}$ & $\mathbf{8 7 . 1}$ \\
MUC & P_3U & 78.1 & 59.3 & 67.4 & 76.2 & 54.8 & 63.7 & 76.1 & 55.3 & 64.1 \\
CEAFm & & 74.4 & 59.9 & 66.4 & $\mathbf{7 5 . 9}$ & 59.6 & $\mathbf{6 6 . 8}$ & $\mathbf{7 5 . 4}$ & $\mathbf{6 0 . 3}$ & $\mathbf{6 7 . 0}$ \\
$B^{3}$ & & 83.0 & 83.0 & 83.0 & $\mathbf{8 3 . 4}$ & $\mathbf{8 3 . 4}$ & $\mathbf{8 3 . 4}$ & $\mathbf{8 3 . 5}$ & $\mathbf{8 3 . 5}$ & $\mathbf{8 3 . 5}$ \\
& & 91.8 & 84.6 & 88.1 & $\mathbf{9 2 . 6}$ & 84.5 & $\mathbf{8 8 . 4}$ & $\mathbf{9 2 . 3}$ & $\mathbf{8 4 . 7}$ & $\mathbf{8 8 . 4}$
\end{tabular}

results of RELAXCOR using the mention-pair model, as explained in Section 4, the next three columns are the results of RELAXCOR adding the features of Section 6.2.1, and the final three columns are the scores for RELAXCOR with the constraints of Section 6.2.2. Note that the main improvements are focused around PN_N, CN_P, and CN_N, as expected. Moreover, the global scores also improve, but the global improvements are not statistically significant.

Although there are improvements in our target classes (PN_N, CN_P, and CN_N), there are some collateral effects that decrease the performance for other classes such as PN_P and P_3U (ungendered pronouns: it). The latter is a strong decrease and, given that the class $P_{\_} 3 \mathrm{U}$ represents $18 \%$ of the total coreferent mentions, this affects the global results. This decrease in pronoun classification performance is related to the balance value learned in the development process. One possible solution would be to have a different balance value depending on the class.

Another phenomenon to take into account in the case of RELAXCOR + features is that the improvement in global scores is in precision but not in recall. This is because the development process is optimizing scores for the CEAF measure, which encourages precision more than recall compared with the MUC scorer.

The improvements achieved seem too few given the necessary effort to extract the knowledge. In general terms, we have found that, although performance is slightly improved on average, few new coreference relations are found, taking into account the expected potential for improvement. Moreover, some of these new relations do not change the final output and, even worse, many of them are incorrect. In addition, some coreferences that were correctly solved before this process are now incorrectly classified. In particular, the recall of ungendered pronouns has decreased considerably.

Finally, it is interesting to remark that these improvements are achieved thanks to a reduced number of mentions in test documents that end up having an actual Wikipediainfluenced constraint (e.g., fewer than $1 \%$ of the mentions in features model). Thus, better extraction procedures or a knowledge source more suitable for entities appearing in the target documents should yield larger improvements. 
More details on the extraction process and a detailed error analysis can be found in Sapena (2012). The error analysis shows how the extracted knowledge was often redundant (i.e., used only in cases where the algorithm already produced the right answer) or noisy (due to errors in the entity disambiguation or information extraction steps). Thus, we think that the experiments show that RELAXCOR is able to incorporate world knowledge into the resolution model in an easy and natural way, and that further work is required on acquiring more accurate and useful knowledge to feed the coreference resolution process.

\section{Conclusions}

In this work, we defined an approach based on constraint satisfaction that represents the problem in a hypergraph and solves it by relaxation labeling, reducing coreference resolution to a hypergraph partitioning problem under a set of constraints. Our approach manages mention-pair and entity-mention models at the same time, and is able to introduce new information by adding as many constraints as necessary. Furthermore, our approach overcomes the weaknesses of previous approaches in state-of-the-art systems, such as linking contradictions, classifications without context, and a lack of information in evaluating pairs.

The presented system, RELAXCOR, achieved state-of-the-art results using only the mention-pair model without new knowledge. Moreover, experiments with the entitymention model showed how it is able to introduce knowledge in a constructive way.

Although it is clearly necessary to incorporate world knowledge to move forward in the field of coreference resolution, the process required to introduce such information in a constructive way has not yet been found. In this work, we tested a methodology that identified the real-world entities referred to in a document, extracted information about them from Wikipedia, and then incorporated this information in two different ways in the model. It seems that neither of the two forms work very well, however, and that the results and errors are in the same direction: The slight improvement of the few new relationships is offset by the added noise. Other state-of-the-art systems have better improvements than ours (Ponzetto and Strube 2006; Uryupina et al. 2011; Rahman and $\mathrm{Ng}$ 2011a), but these also seem too modest given the large amount of information used and the room for improvement outlined in the Introduction.

The problem seems to lie with the extracted information rather than the model used to incorporate it. The extracted information is biased in favor of the more famous and popular entities (those in Wikipedia, and having larger entries). This causes the system to find more information about these entities, including false positives, and causes an imbalance against entities with little or no information in Wikipedia. Moreover, it is not possible to use negative information in the absence of complete information.

Therefore, we believe that research in this field should focus on the extraction of more reliable and concise information, so that the information added, no matter how minimal, should always be constructive and avoid false positives. On the other hand, we would need to find some process of reasoning to expand the scope of the information obtained using logic and common sense. Only then could the full potential of the knowledge base be exploited.

\section{Acknowledgments}

This research was supported by the Spanish Science and Innovation Ministry, via the KNOW2 project (TIN2009-14715-C04-04) and from the European Community's Seventh Framework Programme (FP7/2007-2013) under grant agreement number 247762 (FAUST). 


\section{References}

Aone, C. and S. W. Bennett. 1995. Evaluating automated and manual acquisition of anaphora resolution strategies. In Proceedings of the Annual Meeting of the Association for Computational Linguistics (ACL 1995), pages 122-129.

Atserias, J. 2006. Towards Robustness in Natural Language Understanding. Ph.D. thesis, Departamento de Lenguajes y Sistemas Informáticos, Euskal Herriko Unibertsitatea, Donosti, Spain.

Azzam, S., K. Humphreys, and R. Gaizauskas. 1999. Using coreference chains for text summarization. In Proceedings of the Workshop on Coreference and its Applications, pages 77-84, Stroudsburg, PA.

Bagga, A. and B. Baldwin. 1998. Algorithms for scoring coreference chains. In Proceedings of the Linguistic Coreference Workshop at LREC, pages 563-566, Granada.

Bean, D., E. Riloff, S. Dumais, D. Marcu, and S. Roukos. 2004. Unsupervised learning of contextual role knowledge for coreference resolution. In Proceedings of the Annual Conference of the North American Chapter of the Association for Computational Linguistics (NAACL-HLT 2004), pages 297-304, Boston, MA.

Bengtson, E. and D. Roth. 2008. Understanding the value of features for coreference resolution. In Proceedings of the Conference on Empirical Methods in Natural Language Processing (EMNLP 2008), pages 294-303, Waikiki, HI.

Cai, J. and M. Strube. 2010. End-to-end coreference resolution via hypergraph partitioning. In Proceedings of the 23rd International Conference on Computational Linguistics, pages 143-151, Beijing.

Cardie, C. and K. Wagstaff. 1999. Noun phrase coreference as clustering. In Proceedings of the 1999 Joint SIGDAT Conference on Empirical Methods in Natural Language Processing and Very Large Corpora (EMNLP-VLC 1999), pages 82-89, College Park, MD.

Collins, M. 1999. Head-Driven Statistical Models for Natural Language Parsing. Ph.D. thesis, University of Pennsylvania. Culotta, A., M. Wick, and A. McCallum. 2007. First-order probabilistic models for coreference resolution. In Proceedings of the Annual Conference of the North American Chapter of the Association for Computational
Linguistics (NAACL-HLT 2007), pages 81-88, Rochester, NY.

Denis, P. 2007. New Learning Models for Robust Reference Resolution. Ph.D. thesis, University of Texas at Austin.

Denis, P. and J. Baldridge. 2007. Joint determination of anaphoricity and coreference resolution using integer programming. Proceedings of the Annual Conference of the North American Chapter of the Association for Computational Linguistics (NAACL-HLT 2007), pages 236-243, Rochester, NY.

Denis, P. and J. Baldridge. 2008. Specialized models and ranking for coreference resolution. Proceedings of the Conference on Empirical Methods for Natural Language Processing (EMNLP 2008).

Finkel, J. R. and C. D. Manning. 2008. Enforcing transitivity in coreference resolution. In Proceedings of the Annual Meeting of the Association for Computational Linguistics (ACL 2008), pages 45-48, Columbus, $\mathrm{OH}$.

Finley, T. and T. Joachims. 2005. Supervised clustering with support vector machines. ACM International Conference Proceedings Series, 119:217-224.

Haghighi, A. and D. Klein. 2007. Unsupervised coreference resolution in a nonparametric bayesian model. In Proceedings of the Annual Meeting of the Association of Computational Linguistics (ACL 2007), pages 848-855.

Hummel, R. A. and S. W. Zucker. 1983. On the foundations of relaxation labeling processes. IEEE Transactions on Pattern Analysis and Machine Intelligence, 5(3):267-287.

Ji, H., D. Westbrook, and R. Grishman. 2005. Using semantic relations to refine coreference decisions. Proceedings of the Conference on Human Language Technology and Empirical Methods in Natural Language Processing (HLT-EMNLP 2005), pages 17-24, Prague.

Kingsbury, P. and M. Palmer. 2003. Propbank: the next level of treebank. In Proceedings of Treebanks and Lexical Theories, Växjö.

Klenner, M. 2007. Enforcing consistency on coreference sets. In Proceedings of the Conference on Recent Advances in Natural Language Processing (RANLP 2007), pages 323-328, Borovets.

Klenner, M. and É. Ailloud. 2008. Enhancing Coreference Clustering. In Proceedings of the Second Workshop on Anaphora Resolution (WAR II, 2008), pages 31-40, Bergen. 
Lee, H., Y. Peirsman, A. Chang,

N. Chambers, M. Surdeanu, and

D. Jurafsky. 2011. Stanford's multi-pass sieve coreference resolution system at the CoNLL-2011 shared task. In Proceedings of the Fifteenth Conference on Computational Natural Language Learning: Shared Task, pages 28-34, Portland, OR.

Luo, X. 2005. On coreference resolution performance metrics. Proceedings of the Joint Conference on Human Language Technology and Empirical Methods in Natural Language Processing (HLT-EMNLP 2005), pages 25-32, Vancouver.

Luo, X. 2007. Coreference or not: A twin model for coreference resolution. In Proceedings of the Annual Conference of the North American Chapter of the Association for Computational Linguistics (NAACL-HLT 2007), pages 73-80, Rochester, NY.

Luo, X., A. Ittycheriah, H. Jing, N. Kambhatla, and S. Roukos. 2004. A mention-synchronous coreference resolution algorithm based on the bell tree. In Proceedings of the Annual Meeting of the Association for Computational Linguistics (ACL 2004), pages 135-142, Barcelona.

Màrquez, L., L. Padró, and H. Rodríguez. 2000. A machine learning approach for POS tagging. Machine Learning Journal, 39(1):59-91.

Màrquez, L., M. Recasens, and E. Sapena. 2012. Coreference resolution: An empirical study based on SemEval-2010 shared task 1. Journal on Language Resources and Evaluation, Special Issue on SemEval-2010. doi:10.1007/s510579-012-9194-z.

McCallum, A. and B. Wellner. 2005. Conditional models of identity uncertainty with application to noun coreference. Advances in Neural Information Processing Systems, 17:905-912.

McCarthy, J. F. and W. G. Lehnert. 1995. Using decision trees for coreference resolution. Proceedings of the Fourteenth International Conference on Artificial Intelligence, pages 1,050-1,055.

Mitkov, Ruslan. 2002. Anaphora Resolution. Longman.

Morton, T. S. 2000. Using coreference in question answering. NIST Special Publication SP, pages 685-688.

$\mathrm{Ng}, \mathrm{V} .2005$. Machine learning for coreference resolution: From local classification to global ranking. In Proceedings of the Annual Meeting of the Association for Computational Linguistics (ACL 2005), pages 157-164, Ann Arbor, MI.
$\mathrm{Ng}$, V. 2007. Shallow semantics for coreference resolution. In Proceedings of the International Joint Conference on Artificial Intelligence (IJCAI 2007), pages 1,689-1,694, Hyderabad.

$\mathrm{Ng}$, V. 2008. Unsupervised models for coreference resolution. In Proceedings of the Conference on Empirical Methods in Natural Language Processing (EMNLP 2008), pages 640-649, Waikiki, HI.

$\mathrm{Ng}$, V. 2009. Graph-cut-based anaphoricity determination for coreference resolution. In Proceedings of the Annual Conference of the North American Chapter of the Association for Computational Linguistics (ACL 2009), pages 575-583, Suntec.

Ng, V. 2010. Supervised noun phrase coreference research: The first fifteen years. In Proceedings of the Annual Meeting of the Association for Computational Linguistics (ACL 2010), pages 1,396-1,411, Uppsala.

Ng, V. and C. Cardie. 2002. Improving machine learning approaches to coreference resolution. In Proceedings of the Annual Meeting of the Association for Computational Linguistics (ACL 2002), pages 104-111, Philadelphia, PA.

Nicolae, C. and G. Nicolae. 2006. Best Cut: A graph algorithm for coreference resolution. Proceedings of the Conference on Empirical Methods for Natural Language Processing (EMNLP 2006), pages 275-283, Sydney.

NIST, US. 2003. The ACE 2003 Evaluation Plan. US National Institute for Standards and Technology (NIST), pages 1-8.

Padró, L. 1998. A Hybrid Environment for Syntax-Semantic Tagging. Ph.D. thesis, Departamento de Llenguatges i Sistemes Informàics, Universitat Politécnica de Catalunya.

Pelillo, M. 1997. The dynamics of nonlinear relaxation labeling processes. Journal of Mathematical Imaging and Vision, 7(4):309-323.

Peral, J., M. Palomar, and A. Ferrández. 1999. Coreference-oriented interlingual slot structure \& machine translation. In Proceedings of the Workshop on Coreference and its Applications, pages 69-76, Stroudsburg, PA.

Ponzetto, S. P. and M. Strube. 2006. Exploiting semantic role labeling, WordNet and Wikipedia for coreference resolution. In Proceedings of the Human Language Technology Conference of the North American Chapter of the Association of Computational 
Linguistics (NAACL 2006), pages 192-199, New York, NY.

Poon, H. and P. Domingos. 2008. Joint unsupervised coreference resolution with Markov Logic. In Proceedings of the Conference on Empirical Methods in Natural Language Processing (EMNLP 2008), pages 650-659, Waikiki, HI

Popescu, A. M. and O. Etzioni. 2005. Extracting product features and opinions from reviews. In Proceedings of the Conference on Human Language Technology and Empirical Methods in Natural Language Processing (HLT-EMNLP 2005), pages 339-346, Vancouver.

Pradhan, S., A. Moschitti, N. Xue, O. Uryupina, and Y. Zhang. 2012. CoNLL-2012 shared task: Modeling multilingual unrestricted coreference in OntoNotes. In Proceedings of the Conference on Computational Natural Language Learning (CoNLL 2012), pages 1-40, Jeju Island.

Pradhan, S., L. Ramshaw, M. Marcus, M. Palmer, R. Weischedel, and N. Xue. 2011. CoNLL-2011 shared task: Modeling unrestricted coreference in OntoNotes. In Proceedings of the Conference on Computational Natural Language Learning (CoNLL 2011), pages 1-27, Portland, OR.

Pradhan, S. S., L. Ramshaw, R. Weischedel, J. MacBride, and L. Micciulla. 2007. Unrestricted coreference: Identifying entities and events in OntoNotes. In Proceedings of the International Conference on Semantic Computing (ICSC 2007), pages $446-453$.

Quinlan, J. R. 1993. C4.5: Programs for Machine Learning. Morgan Kaufmann.

Rahman, A. and V. Ng. 2011a. Coreference resolution with world knowledge. In Proceedings of the Annual Meeting of the Association for Computational Linguistics (ACL 2011), pages 814-824, Portland, OR.

Rahman, A. and V. Ng. 2011b. Narrowing the modeling gap: A cluster-ranking approach to coreference resolution. Journal of Artificial Intelligence Research, 40(1):469-521.

Recasens, M., L. Màrquez, E. Sapena, M. A. Martí, M. Taulé, V. Hoste, M. Poesio, and Y. Versley. 2010. SemEval-2010 Task 1: Coreference resolution in multiple languages. In Proceedings of the International Workshop on Semantic Evaluations (SemEval-2010), pages 1-8, Uppsala.

Rosenfeld, R., R. A. Hummel, and S. W. Zucker. 1976. Scene labelling by relaxation operations. IEEE Transactions on Systems, Man and Cybernetics, 6(6):420-433.

Rounds, E. M. 1980. A combined nonparametric approach to feature selection and binary decision tree design. Pattern Recognition, 12(5):313-317.

Safavian, S. R. and D. Landgrebe. 1991. A survey of decision tree classifier methodology. IEEE Transactions on Systems, Man and Cybernetics, 21(3):660-674.

Sapena, E. 2012. A Constraint-Based Hypergraph Partitioning Approach to Coreference Resolution. Ph.D. thesis, Universitat Politecnica de Catalunya.

Sapena, E., L. Padró, and J. Turmo. 2010a. A global relaxation labeling approach to coreference resolution. In Proceedings of the International Conference on Computational Linguistics (COLING 2010), pages 1,086-1,094, Beijing.

Sapena, E., L. Padró, and J. Turmo. 2010b. RelaxCor: A global relaxation labeling approach to coreference resolution. In Proceedings of the ACL Workshop on Semantic Evaluations (SemEval-2010), pages 88-91, Uppsala.

Sapena, E., L. Padró, and J. Turmo. 2011. RelaxCor participation in CoNLL shared task on coreference resolution. In Proceedings of the Fifteenth Conference on Computational Natural Language Learning: Shared Task, pages 35-39, Portland, OR.

Soon, W. M., H. T. Ng, and D. C. Y. Lim. 2001. A machine learning approach to coreference resolution of noun phrases. Computational Linguistics, 27(4):521-544.

Stoyanov, V., N. Gilbert, C. Cardie, and E. Riloff. 2009. Conundrums in noun phrase coreference resolution: Making sense of the state-of-the-art. In Proceedings of the Joint Conference of the Annual Meeting of the ACL and the International Joint Conference on Natural Language Processing (ACL-IJCNLP 2009), pages 656-664, Suntec.

Torras, C. 1989. Relaxation and neural learning: Points of convergence and divergence. Journal of Parallel and Distributed Computing, 6:217-244.

Uryupina, O. 2009. Detecting anaphoricity and antecedenthood for coreference resolution. Procesamiento del Lenguaje Natural, pages 113-120.

Uryupina, O., M. Poesio, C. Giuliano, and K. Tymoshenko. 2011. Disambiguation and filtering methods in using Web knowledge for coreference resolution. In Proceedings of 
the International Florida Artificial Intelligence Research Society Conference, pages 317-322,

Palm Beach, FL.

Vilain, M., J. Burger, J. Aberdeen, D. Connolly, and L. Hirschman. 1995. A model-theoretic coreference scoring scheme. In Proceedings of the Message Understanding Conference (MUC-6), pages 45-52, Arlington, VA.

Yang, X., J. Su, J. Lang, C. L. Tan, T. Liu, and S. Li. 2008. An entity-mention model for coreference resolution with inductive logic programming. In Proceedings of the Annual Meeting of the Association for Computational Linguistics (ACL 2008), pages 843-851, Columbus, $\mathrm{OH}$.

Yang, X., J. Su, and C. L. Tan. 2006.

Kernel-based pronoun resolution with structured syntactic knowledge. Proceedings of the 21st International Conference on Computational Linguistics and the 44th Annual Meeting of the Association for Computational Linguistics (COLING-ACL 2006), pages 41-48, Sydney.

Yang, X., J. Su, G. Zhou, and C. L. Tan. 2004. An NP-cluster based approach to coreference resolution. In Proceedings of the International Conference on Computational Linguistics (COLING 2004), pages 226-232, Geneva.

Yang, X., G. Zhou, J. Su, and C. L. Tan. 2003. Coreference resolution using competition learning approach. In Proceedings of the Annual Meeting of the Association for Computational Linguistics (ACL 2003), pages 176-183, Sapporo. 